\title{
Luisa Wendel
}

Welche Grundrechte führen zum Erfolg?

Eine quantitative, korpusgestützte Untersuchung anhand von

Entscheidungen des Bundesverfassungsgerichts

- Zweitveröffentlichung -

Erstveröffentlichung in

Juristenzeitung 2020, S. 668-679

(DOI: 10.1628/jz-2020-0128) 


\title{
Welche Grundrechte führen zum Erfolg?
}

\author{
Eine quantitative, korpusgestützte Untersuchung anhand von Entscheidungen des \\ Bundesverfassungsgerichts
}

Es existieren keine offiziellen Statistiken darüber, welche Grundrechte besonders häufig verletzt sind und welche besonders häufig in Verfassungsbeschwerden gerügt werden. Aufgrund des Umfangs des verfassungsgerichtlichen Entscheidungskorpus ist eine manuelle Zählung heutzutage nicht mehr möglich. Dieser Beitrag versucht, mit computergestützten Methoden Antworten auf diese und verwandte Fragen zu finden.

\section{Forschungsfragen und Literatur}

Quantitative Methoden finden zunehmend auch in der deutschsprachigen Rechtswissenschaft Verbreitung. ${ }^{1}$ Die dafür auszuwertenden Daten können wirtschaftliche, politische ${ }^{2}$ oder soziologische Merkmale aufgreifen oder aus juristischen Texten selbst gewonnen werden ${ }^{3}$. Letzteres geht häufig mit dem Einsatz von Methoden aus der Korpuslinguistik einher. ${ }^{4}$ Der Rückgriff auf quantitativempirische Methoden soll dazu beitragen, die Generierung von rechtswissenschaftlichen Erkenntnissen transparenter zu gestalten und auf anekdotischem Wissen aufbauende Theorien zu überprüfen. Die quantitative Auswertung von Texten mit maschinellen Mitteln ermöglicht darüber hinaus, Erkenntnisse aus einer Textmenge zu gewinnen, die zu groß ist, um jeden Text einzeln zu lesen, oder Strukturen zu erkennen, die einem menschlichen Leser auf den ersten Blick entgehen.

Wir werden im Folgenden mit computergestützten Methoden anhand eines Korpus der Entscheidungen des Bundesverfassungsgerichts der Frage nachgehen, welche Grundrechtsverletzungen in Verfassungsbeschwerden besonders häufig festgestellt werden, welche Grundrechte besonders häufig gerügt werden und wie sich diese Werte zueinander verhalten. Diese Frage eignet sich für eine korpusgestützte Untersuchung, weil das dazu bisher verfügbare Datenmaterial uneinheitlich und nicht aktuell ist und eine Lektüre aller aktuell verfügbaren Entscheidungen einen menschlichen Leser überfordern dürfte.

* Die Autorin ist Wissenschaftliche Mitarbeiterin im Forschungsprojekt Leibniz Linguistic Research into Constitutional Law (L.L.Con.) unter der Leitung von Prof. Dr. Christoph Möllers, Humboldt-Universität zu Berlin, und gefördert aus Mitteln des Leibniz-Preises der DFG. Für die Unterstützung bei der Konzeption, Datenerhebung und -auswertung dankt sie Louis Rolfes, Anna Shadrova und Dr. Alexander Tischbirek, für wertvolle Anregungen außerdem Prof. Dr. Christoph Möllers, Dr. Jens Frankenreiter und Ali Ighreiz.

1 Zu grundlegenden Problemen quantitativer Methoden in der Rechtswissenschaft: Coupette/Fleckner JZ 2018 , 379.

2 Beispielsweise die Parteinähe von Richtern: Engst et. al. JZ 2017, 816.

3 Beispielsweise Vogel/Christensen Rechtstheorie 2013, 29.

4 Zum Begriff Lemnitzer/Zinsmeister, Korpuslinguistik - Eine Einführung, 3. Aufl. 2015, S. 14. 


\section{Stand der Forschung}

Der Internetseite des Bundesverfassungsgerichts ist zu entnehmen, dass im Zeitraum von 1951 bis 2017 ca. 232.000 Verfahren vor dem Bundesverfassungsgerichts anhängig wurden, davon ca. 224.000 Verfassungsbeschwerden, ${ }^{5}$ von denen ca. 5.000 erfolgreich waren. ${ }^{6}$ Weiterhin kann sich der Leser darüber informieren, wie viele Bundes- und Landesnormen und wie viele Gerichtsentscheidungen beanstandet wurden. Eine offizielle Statistik des Bundesverfassungsgerichts über die dabei gerügten oder als verletzt angesehenen Grundrechtsnormen gibt es so weit ersichtlich jedoch nicht. ${ }^{7}$ Nach allgemeiner Ansicht ist das Recht auf rechtliches Gehör aus Art. 103 Abs. 1 GG nicht nur das am häufigsten als verletzt gerügte, sondern auch das tatsächlich am häufigsten verletzte Grundrecht. Auf eine Angabe konkreter Zahlen wird dabei jedoch oft verzichtet. ${ }^{8}$ Wenn Zahlen angegeben werden, so schwanken diese stark und nicht immer ist ihre Quelle erkennbar. Dazu einige Beispiele:

Im Jahr 1969 sollen 512 von 759 eingegangen Verfassungsbeschwerden (ca. 67 \%) Art. 103 Abs. 1 GG betroffen haben. ${ }^{9}$ Für die 1980er-Jahre wird von $45 \%$ gesprochen ${ }^{10}$ aber gelegentlich auch von 75 \%. ${ }^{11}$ Dreißig Prozent der erfolgreichen Verfassungsbeschwerden sollte eine Verletzung des Rechts auf rechtliches Gehör zugrundeliegen. ${ }^{12}$ Jedoch wird auch eingeräumt, dass statistische Erhebungen zum Anteil an den erfolgreichen Verfassungsbeschwerden fehlen. ${ }^{13}$ Auch im Jahr 1995 sollen noch 45 \% der auf den Ersten Senat entfallenden Verfassungsbeschwerden Art. 103 Abs. 1 GG betroffen haben. ${ }^{14}$

Nach Nolte soll, unter Bezug auf eine unveröffentlichte Statistik des Bundesverfassungsgerichts, die Zahl der vorrangig auf Art. 103 Abs. 1 GG gestützten Verfassungsbeschwerden im Zeitraum von 2005 bis 2009 gegenüber dem Zeitraum 1996 bis 2004 von durchschnittlich 395 pro Jahr auf durchschnittlich 260 pro Jahr gesunken sein. ${ }^{15}$ Remmert weist darauf hin, dass die Annahmen zu Art.

5 Bundesverfassungsgericht, Verfahren seit 7. September 1951 bis 31 . Dezember 2017, https://www.bundesverfassungsgericht.de/DE/Verfahren/Jahresstatistiken/2017/gb2017/A-I-

1.pdf? blob=publicationFile\&v=2 (letzter Abruf am 3. Dezember 2019).

6 Siehe für eine Aufschlüsselung nach Jahr auch: Bundesverfassungsgericht, Anteil der stattgegebenen an den $\begin{array}{lllll}\text { entschiedenen } & \text { Verfassungsbeschwerdeverfahren } & \text { pro } & \text { Jahr } & \text { seit }\end{array}$ https://www.bundesverfassungsgericht.de/DE/Verfahren/Jahresstatistiken/2017/gb2017/A-IV-

2.pdf? blob=publicationFile\&v=2 (letzter Abruf am 3. Dezember 2019).

7 So auch Graßhof, in: Merten/Papier (Hrsg.), HdbGR V, § 133 Fn. 30; Remmert, in: Maunz/Dürig, GG, Lfg. 78 September 2016, Art. 103 Abs. 1 Rn. 7 Fn. 5.

8 Vgl. Graßhof, in: Merten/Papier (Fn. 7) § 133 Rn. 9; Kunig, in: v. Münch/Kunig, 6. Aufl. 2012, Art. 103 Abs. 1 Rn. 1; Redeker NJW 2003, 2956, 2957.

9 Endemann NJW 1969, 1197.

10 BT-Drs. 10/2951, S. 6. Mit Bezug auf BR-Drs. 614/84, S. 8 aber unter Hinweis darauf, dass eine detaillierte statistische Auszählung nicht vorläge, die angegebenen Zahlen vielmehr auf „langjährigen Erfahrungssätzen“ beruhten Zierlein DVBl. 1989, 1169 Fn. 10. Aktueller auch Schulze-Fielitz, in: Dreier (Hrsg.), GG, Bd. III, 2018, Art. 103 I, Rn. 15; Höfling, in: Friauf/Höfling (Hrsg.), Berliner Kommentar, Bd. 5, 2009, Art. 103 Rn. 5.

11 Ohne Beleg: Hübsch DRiZ 1980, 140 und Seetzen NJW 1982, 2337; unter Verweis auf Hübsch: Braun NJW 1981 , 425. Zur Unsicherheit dieser Angaben auch Waldner, Der Anspruch auf rechtliches Gehör, Köln 1989, 1.

12 Seetzen NJW 1982, $2337 \mathrm{f}$.

13 Graßhof, in: Merten/Papier (Fn. 7) § 133 Rn. 32; Zierlein DVBl. 1989, 1169 bezieht sich auf eine unveröffentlichte Zusammenstellung der Geschäftsstellen des Bundesverfassungsgerichts von Ende März 1989.

14 BMJ, Entlastung des Bundesverfassungsgerichts - Bericht der vom Bundesminister der Justiz eingesetzten Kommission, 1998, S. 63.

15 Nolte, in: v. Mangoldt/Klein/Starck, GG, Bd. 3, 2010, Art. 103 Abs. 1 Fn. 4. 
103 Abs. 1 GG zwar plausibel, aber nicht belastbar belegt seien; auch die Vermutung, dass die Zahl der auf Art. 103 Abs. 1 GG gestützten Verfassungsbeschwerden seit 2005 zurückginge, ließe sich „mit vertretbarem Aufwand nicht näher verifizieren“. ${ }^{16}$ Doch selbst wenn Art. 103 Abs. 1 GG die unangefochtene Nummer 1 der verletzten Grundrechtsnormen ist, welche Grundrechte folgen auf den Rängen 2 ff.? Und gab es diesbezüglich Veränderungen im Laufe der Zeit?

In der Vergangenheit gab es durchaus Versuche, Statistiken über die Betroffenheit oder Verletzung der einzelnen Grundrechte, nicht nur von Art. 103 Abs. 1 GG, zu erstellen. Eine Auswertung der ersten 40 Bände der amtlichen Sammlung findet sich bei Peters. ${ }^{17}$ Von 269 begründeten Verfassungsbeschwerden betrafen 83 Art. 103 GG, gefolgt von Art. 3 GG (45 mal), Art. 2 GG (38 mal) und Art. 12 GG (24 mal), wobei jeweils weiter nach Absätzen und verbundenen Artikeln differenziert wird. ${ }^{18}$ Der Autor vermutet, dass trotz dieser hohen absoluten Zahlen die Erfolgsquote von Verfassungsbeschwerden zu diesen Grundrechten eher gering sein könnte. ${ }^{19}$

Nach einer Zusammenstellung der Bände 1 bis 67 der amtlichen Sammlung von Schumann aus dem Jahr 1985 war Art. 103 Abs. 1 GG in mehr als der Hälfte der Verfahren (95 von 187, ,zumeist Verfassungsbeschwerden“) als Prüfungsmaßstab herangezogen worden, ${ }^{20}$ wobei der Autor darauf hinweist, dass die Entscheidungen der Dreierausschüsse gem. § 93a Abs. 3 BVerfGG a.F. sowie nach einem Belehrungsschreiben zurückgenommene Verfassungsbeschwerden nicht erfasst sind. Auch soll der Anteil der Entscheidungen zu Art. 103 Abs. 1 GG an der Gesamtzahl der Entscheidungen im Laufe der Zeit zugenommen haben. ${ }^{21}$ Es folgen Art. 3 Abs. 1 GG (21 Entscheidungen) und das Rechtsstaatsprinzip (15 Entscheidungen).

Von Höfling und Rixen stammt eine Übersicht der Kammerbeschlüsse aus den Jahren 1986 bis 1998, die nach den Kategorien „Eingriffsbegrenzungsjudikatur“ und „Rechtsschutzeffektuierungsjudikatur“, und weiter nach einzelnen Grundrechten, unterteilt ist und insgesamt eher qualitativer als quantitativer Natur ist. ${ }^{22}$ Berücksichtigt wurden 547 von 1903 Entscheidungen, die im Falle der Betroffenheit mehrerer Rechte eindeutig einer Kategorie zugeordnet wurden. ${ }^{23}$ Eine auf das Jahr 2011 begrenzte Auswertung wurde von Engel durchgeführt, in diesem Jahr beschäftigte sich das Bundesverfassungsgericht häufiger mit Art. 3 Abs. 1 GG, Art. 2 Abs. 1 GG oder Art. 14 Abs. 1 GG als mit Art. 103 Abs. 1 GG. ${ }^{24}$

Die vorhandenen (umfassenden) quantitativen Auswertungen unterscheiden sich also nicht nur methodisch, sondern enden auch ungefähr in dem Moment, in dem die Veröffentlichung der begründeten Kammerentscheidungen auf der Internetseite des Bundesverfassungsgerichts beginnt (im Jahr 1998) und somit die vorhandene Datenmenge einerseits umfangreicher, andererseits auch

16 Remmert, in: Maunz/Dürig (Fn. 7) Art. 103 Abs. 1 Rn. 7.

17 Peters MDR 1976, 447-452.

18 Peters MDR 1976, 447, 452.

19 Peters MDR 1976, 447, 452.

20 Schumann NJW 1985, 1134, 1135.

21 Schumann NJW 1985, 1134, 1135.

22 Höfling/Rixen AöR 2000, 428-476 und dies. AöR 2000, 613-647.

23 Höfling/Rixen AöR 2000, 428, 435.

24 Engel, in: Jestaedt/Lepsius (Hrsg.), Verhältnismäßigkeit - Zur Tragfähigkeit eines verfassungsrechtlichen Schlüsselkonzepts, 2015, S. 97, 102. 
leichter automatisch durchsuchbar wird. An dieser Stelle möchte die vorliegende Untersuchung einsetzen.

\section{Forschungsfragen}

Uns interessieren dabei folgende Fragen:

1. Welche Grundrechte sind in erfolgreichen Verfassungsbeschwerden „absolut“ am häufigsten verletzt?

2. Welche Grundrechte werden besonders häufig von den Beschwerdeführerinnen gerügt?

3. Wie verändern sich diese Häufigkeiten im Laufe der Zeit? Insbesondere: Verändern sich die Zahlen hinsichtlich des Rechts auf rechtliches Gehör nach Einführung der Anhörungsrüge?

4. Welche Grundrechte sind „relativ“ gesehen besonders erfolgreich, d.h. bei welchen Grundrechten ist der Anteil der tatsächlichen Verletzungen bezogen auf die Rügen insgesamt besonders hoch?

Dabei werden wir deskriptiv und explorativ vorgehen. Die deskriptive Statistik umfasst Methoden zur Beschreibung und Darstellung von Stichprobendaten. ${ }^{25}$ Aus Stichprobendaten auf die gesamte Population zu schließen, ist dagegen Gegenstand der Inferenzstatistik, ${ }^{26}$ die hier nicht zum Einsatz kommen soll. Methoden der explorativen Datenanalyse werden eingesetzt, um Anhaltspunkte für weitergehende, bestätigende Analysen zu finden ${ }^{27}$ und um Daten nach Mustern, Auffälligkeiten oder Zusammenhängen zu untersuchen. ${ }^{28}$ Dabei geht es (noch) nicht um die Falsifizierung zuvor formulierter Nullhypothesen. ${ }^{29}$

Nach einer kurzen Darstellung unserer Methode (II.) werden wir unsere Ergebnisse vorstellen (III.), diese interpretieren (IV.) und zusammenfassen (V.).

\section{Methode ${ }^{30}$}

\section{Vorbereitung des Korpus}

Das für die Untersuchung verwendete Korpus besteht aus 9.261 Entscheidungen des Bundesverfassungsgerichts, von denen 6.579 von der Internetseite des Bundesverfassungsgerichts stammen (Zeitraum vom 1.1.1998 bis 31.12.2017) und durch 2.682 Dokumente ${ }^{31}$ aus der Amtlichen Sammlung (bis 31.12.1997) ergänzt werden, es ist also auf veröffentlichte Entscheidungen beschränkt. Dies entspricht der Vorgehensweise in früheren Untersuchungen, die in der Regel

25 Meindl, Methodik für Linguisten - Eine Einführung in Statistik und Versuchsplanung, 2011, S. 67; Schäfer, Methodenlehre und Statistik - Einführung in Datenerhebung, deskriptive Statistik und Inferenzstatistik, Wiesbaden 2016, S. 47.

26 Meindl (Fn. 25), S. 132.

27 Tukey, Exploratory Data Analysis, 1977, S. 3.

28 Schäfer (Fn. 25), S. 83, 255.

29 Meindl (Fn. 25), S. 96. Allgemein zur Gewinnung von Hypothesen für quantitative Untersuchungen von Gerichtsentscheidungen: Möllers/Tischbirek, From Data to Dogma. How to find a legal argument?, Manuskript 2019.

30 Eine ausführliche Beschreibung der Vorgehensweise und die Begriffskataloge sind im Anhang zu diesem Beitrag zu finden (DOI: 10.5281/zenodo.3560824).

31 Darunter auch 4 Anhänge bzw. getrennte Leitsätze. 
ebenfalls nur veröffentlichte Untersuchungen berücksichtigten, soweit ihre Quellen überhaupt erkennbar sind ${ }^{32}$. Wir gehen davon aus, dass alle Entscheidungen mit Begründung ab 1998 auf der Internetseite des Bundesverfassungsgerichts veröffentlicht wurden. ${ }^{33}$ Die Dokumente wurden mit der Open Source Software GATE ${ }^{34}$ annotiert, d.h. relevante Textmuster wurden gekennzeichnet (Abb. 1). ${ }^{35}$

Wir gehen grundsätzlich davon aus, dass erfolgreiche Verfassungsbeschwerden (mindestens) einen Tenorsatz enthalten, in dem festgestellt wird, welche Grundrechtsnorm(en) durch den angegriffenen Akt der öffentlichen Gewalt verletzt ist/sind (§ 95 Abs. 1 S. 1 BVerfGG). Unser Ziel ist, diese Grundrechtsnormen aufzuschlüsseln. Wir verwenden daher eine Annotationskategorie, die einen solchen Tenorsatz kennzeichnet und eine Annotationskategorie, die das Zitat einer Grundrechtsnorm markiert.

Herauszufinden, welche Grundrechte gerügt wurden, ist schwieriger. Wir wissen nicht, was in den Schriftsätzen steht, sondern nur, was im Tatbestand des Urteils als Rechtsansichten der Beschwerdeführerinnen (und anderer Beteiligter) geschildert wird. Die Wiedergabe der Rechtsansichten ist weniger standardisiert als der Tenor, viele verschiedene Formulierungen kommen in Betracht. Gerade in Kammerentscheidungen fehlt die Wiedergabe des Vortrags des Beschwerdeführers mitunter auch ganz. Durch die wiederholte Auswertung von Stichproben haben wir einen Katalog mit 130 typischen Formulierungen („rügt die Beschwerdeführerin“ usw.) erstellt, die wir als Anhaltspunkte für eine Rüge ansehen, weitere Kombinationen von Formulierungen werden in den Annotationsregeln berücksichtigt. In manchen Entscheidungen werden die gerügten Grundrechte nicht als Grundgesetzzitate genannt, sondern nur mit Worten umschrieben. ${ }^{36}$ Auch dafür haben wir einen Begriffskatalog erstellt, mit dem wir den Wörtern konkrete Zitate zuordnen. ${ }^{37}$ Eine Fehlerabschätzung ergab, dass mit diesem Ansatz für ca. 86 \% der Verfassungsbeschwerden, die eine explizite Rüge enthalten, die gerügten Grundrechte gefunden werden.

32 Vgl. oben Fn. 11.

33 Engel, in: Jestaedt/Lepsius (Fn. 24), S. 97, 128, weist allerdings darauf hin, dass die Veröffentlichungspraxis bei offensichtlich unzulässigen, unbegründeten oder begründeten Kammerentscheidungen uneinheitlich sei.

34 General Architecture for text engineering, https://gate.ac.uk/, Cunningham et. al., Getting More Out of Biomedical Documents with GATE's Full Lifecycle Open Source Text Analytics, PLoS Comput Biol 9(2) (2013): e1002854. doi:10.1371/journal.pcbi.1002854. Für die Erstellung der Annotationsroutinen wurde der GATE Developer verwendet, für die Anwendung auf das gesamte Korpus der GATE Cloud Paralleliser (GCP), https://gate.ac.uk/gcp/ (jeweils letzter Abruf am 3. Dezember 2019).

35 Dabei kommen sogenannte JAPE-Regeln zum Einsatz: https://gate.ac.uk/sale/tao/splitch8.html (letzter Abruf am 3. Dezember 2019).

36 Beispielsweise „,...] wegen Verstoßes gegen den Gleichheitssatz [...], BVerfGE 11, 351, 354.

37 Dieser Katalog ist allerdings eher auf Abwehrrechte ausgelegt, Rügen von Verletzungen von Leistungsrechten sind daher eventuell unterrepräsentiert. 


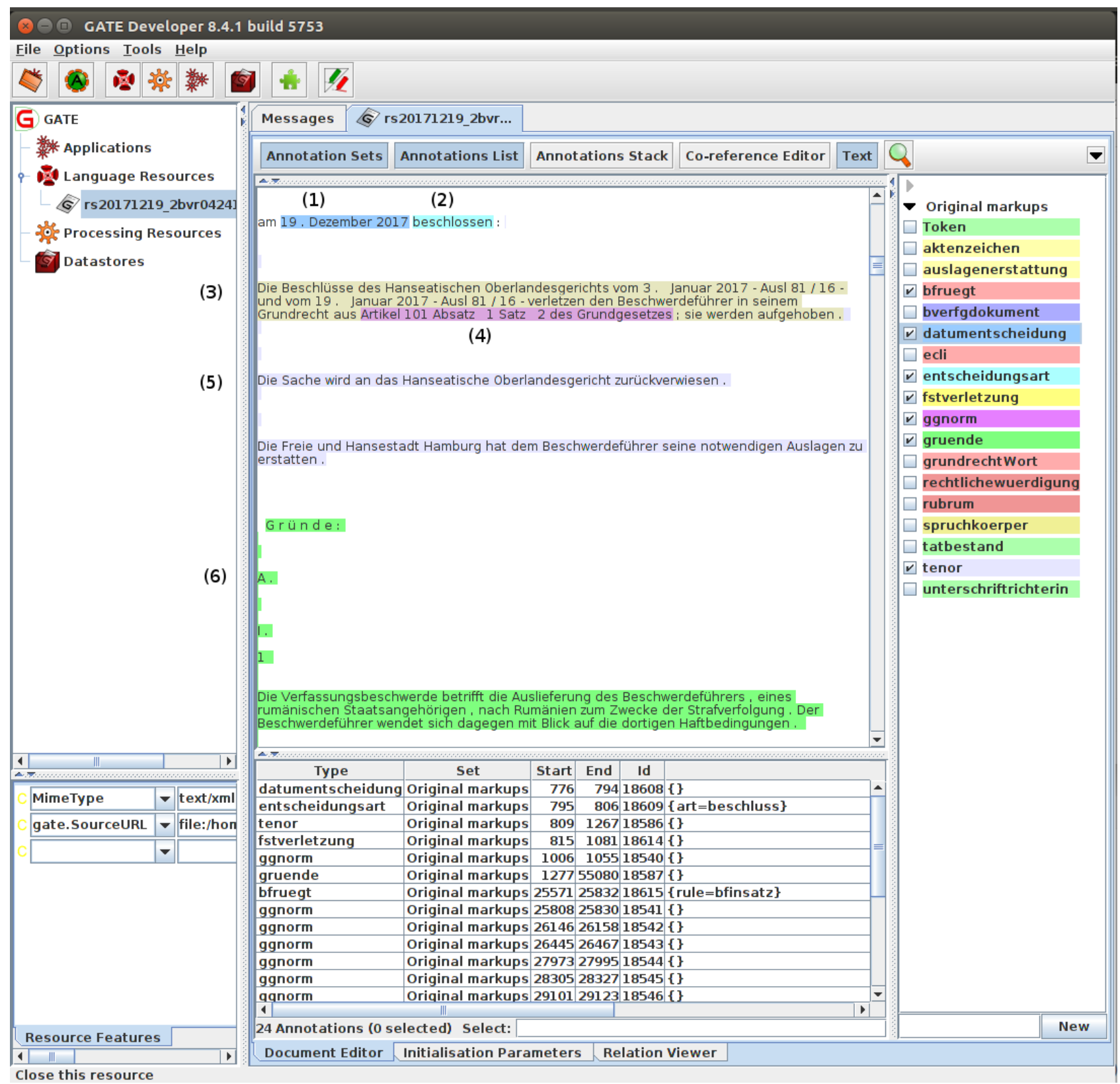

Abbildung 1: Dokument in GATE Developer mit den automatischen Annotationen Datum der Entscheidung (1), Entscheidungsart (2), Feststellung der Verletzung (3), Grundgesetzzitat (4), Tenor (5, einschließlich 3 und 4), Gründe (6).

Mithilfe weiterer Annotationen haben wir eine Tabelle mit Metadaten aller Entscheidungen (Dateiname, Aktenzeichen, Spruchkörper, Entscheidungsdatum, beteiligte Richterinnen usw.) erstellt. Außerdem lassen wir Verfahren erkennen, in denen nur über eine einstweilige Anordnung, Prozesskostenhilfe, ein Ablehnungsgesuch oder den Gegenstandswert entschieden wird, da wir diese nicht untersuchen wollen. 


\section{Datengewinnung}

Die fertig annotierten Texte werden als XML-Dokumente gespeichert und mittels in der Programmiersprache Python ${ }^{38}$ geschriebener Skripte auf das Vorkommen der relevanten Annotationen (Feststellung der Verletzung bzw. Grundrechtsrüge und darin enthaltenes Grundgesetzzitat oder typische Umschreibung eines Grundrechts) durchsucht. Für die Auswertung der tatsächlichen Verletzungen wird eine Tabelle erstellt, die einen Datensatz pro als verletzt bezeichneter Grundgesetznorm innerhalb einer bestimmten Entscheidung enthält. Das bezeichnete Grundrecht wird nach Artikel, Absatz und Satz in mehrere Spalten aufgelöst, damit später eine Auswertung mit oder ohne Angabe von Absatz und Satz möglich ist.

Zitate von Grundrechtsnormen können aber auch durchaus mehrere Absätze und mehrere Sätze enthalten. Angesichts unserer Aufgabenstellung, „Grundrechte zu zählen“, hatten wir hier also die grundsätzliche Frage zu entscheiden, was wir als „ein Grundrecht“ zählen. Wir haben uns dafür entschieden, in einem Datensatz für einen Artikel nur einen Absatz und einen Satz zuzulassen. Ein Zitat wie „Artikel 6 Absatz 1 und Absatz 2 Satz 1“39 wird also in zwei Datensätze mit „6 - 1 - NA“ und „6 - 2 - 1“ aufgeteilt. Die Aufteilung der Zitate auf die Spalten erfolgte überwiegend automatisch, in einigen ungewöhnlichen Fällen manuell. Sofern ein Grundrechtszitat auch auf das Rechtsstaatsprinzip (welches oft ohne Artikel zitiert wird) Bezug nimmt, haben wir dieses, auch wenn es sich aus einer Gesamtschau verschiedener Artikel ergibt, ${ }^{40}$ als Art. 20 Abs. 3 GG codiert.

Eine weitere Grundsatzentscheidung erforderten die ausdrücklichen Verbindungen von Grundrechtsnormen (durch „in Verbindung mit“). Wenn eine Verbindung aus zwei Grundrechten verletzt ist, so ist davon auszugehen, dass dies etwas anderes bedeutet als separate Verletzungen beider Grundrechte. ${ }^{41}$ Die Artikel einzeln zu zählen und die Information über die ausdrückliche Verbindung zu verlieren, würde der Sache daher nicht gerecht. Gleichwohl fehlt eine Kombinationsdogmatik des Bundesverfassungsgerichts. ${ }^{42}$ Eine ausdrückliche Verbindung kann neue Grundrechte schaffen, die nicht ausdrücklich aus dem Grundgesetz zu entnehmen sind ${ }^{43}$ oder bestehende Rechte verstärken. ${ }^{44}$ Grundrechte verschiedener Grundrechtsträger, z.B. spezielle Gleichheitsrechte, ${ }^{45}$ können kombiniert werden, um eine einheitliche Argumentation innerhalb ihrer gemeinsamen Verfassungsbeschwerde oder zwischen Verfassungsbeschwerden und konkreten Normenkontrollen zu ermöglichen. ${ }^{46}$ Eine Verbindung mit dem Rechtsstaatsprinzip kann zur Betonung der verfahrensrechtlichen Seite eines Grundrechts erfolgen, ${ }^{47}$ eine Verknüpfung mit der Menschenwürde zur Begründung staatlicher Pflichten kann dazu dienen, in der Abwägung

38 Python Software Foundation, Python, 2001-2019, https://www.python.org/, dabei wurde insbesondere auf die „Beautiful Soup“-Bibliothek zurückgegriffen: Richardson et. al., Beautiful Soup, 2004-2018, https://www.crummy.com/software/BeautifulSoup/ (jeweils letzter Abruf am 3. Dezember 2019).

39 BVerfG, Beschluss der 2. Kammer des Zweiten Senats vom 30. Januar 2002 - 2 BvR 231/00.

40 Huster/Rux, in: Epping/Hillgruber (Hrsg.), BeckOK GG, 41. Ed., Stand 15.02.2019, Art. 20 Rn. 140.

41 Meinke, In Verbindung mit, 2006, S. 251 (,mehr als zufällige Anhäufungen von grundgesetzlichen Bestimmungen“).

42 Breckwoldt, Grundrechtskombinationen, 2015, S. 6.

43 Augsberg/Augsberg AöR 2007, 539, 542; Breckwoldt (Fn. 42), S. 98; Meinke (Fn. 41) S. 55, 156;

44 Augsberg/Augsberg AöR 2007, 539, 542.

45 Augsberg/Augsberg AöR 2007, 539, 563.

46 Meinke (Fn. 41) S. 32, 127.

47 Meinke (Fn. 41) S. 201. 
entgegenstehenden Rechten aus Art. 1 Abs. 1 zu begegnen. ${ }^{48}$ Verbindungen können einen lückenlosen Grundrechtsschutz sichern, ${ }^{49}$ die Prüfungskompetenzen des BVerfG erweitern ${ }^{50}$ oder als Appel an die Fachgerichte fungieren. ${ }^{51}$ Andererseits kann eine Verbindung auch zur Rücknahme einer früheren Ausdehnung des Schutzbereichs eines Grundrechts dienen. ${ }^{52}$ Eine Verbindung mit dem Sozialstaatsprinzip kann erfolgen, um zu betonen, dass das Sozialstaatsprinzip selbst nicht als eigenständige Anspruchsgrundlage herangezogen werden kann. ${ }^{53}$ Auch kann sich die Bedeutung der Verbindung über die Zeit wandeln. ${ }^{54}$

Allerdings soll die Verwendung von „in Verbindung mit“ mitunter auch nur auf gleichzeitig betroffene Einzelgrundrechte hindeuten. ${ }^{55}$ Weiterhin kommen Grundrechtskombinationen in Betracht, die ohne die Verbindungsformel auskommen. ${ }^{56}$ Beide Fälle lassen sich mit unserer Herangehensweise nicht abgrenzen. Wir haben beschlossen, Verbindungen von zwei Grundrechtsnormen als besondere Ausprägung der Variable „verletztes Grundrecht“ zu berücksichtigen und nicht lediglich die in einer Verbindung genannten Normen einzeln zu zählen, auch wenn nicht jede Verbindung ein neues Grundrecht begründet. Dementsprechend enthält jeder Datensatz weitere Spalten mit Informationen über das Vorliegen einer Verbindung und den Artikel, Absatz und Satz des zweiten Teils der Verbindung (Abb. 2).

\begin{tabular}{|c|c|c|c|c|c|c|c|c|}
\hline dateiname & grundrecht & art & abs & $\mathbf{s}$ & verbindung & art2 & abs2 & $s 2$ \\
\hline rk20171214_2bvr265517.xml & Art. 2 Abs. 2 S. 2 & 2 & 2 & 2 & $2 \mathrm{NA}$ & NA & NA & NA \\
\hline rk20171218_2bvr225917.xml & Art. 19 Abs. 4 S. 1 in Verbindung mit Art. 2 Abs. 2 S. 1 & 19 & 4 & 4 & 1 VERBINDUNG & 2 & 2 & \\
\hline rk20171220_2bvr231217.xml & Art. 19 Abs. 4 & 19 & & $4 \mathrm{NA}$ & NA & NA & NA & NA \\
\hline rk20171220_2bvr255217.xml & Art. 2 Abs. 2 S. 2 & 2 & 2 & \begin{tabular}{|l|l|}
2 \\
\end{tabular} & $2 \mathrm{NA}$ & NA & NA & NA \\
\hline rs19980220_1bvr066194.xml & Art. 5 Abs. 1 S. 2 & 5 & 1 & 1 & 2 NA & NA & NA & NA \\
\hline rs19980324_1bvr013196.xml & Art. 5 Abs. 1 S. 1 & 5 & 1 & 1 & $1 \mathrm{NA}$ & NA & NA & NA \\
\hline rs19980324_1bvr013196.xml & Art. 2 Abs. 1 in Verbindung mit Art. 1 Abs. 1 & 2 & & $1 \mathrm{NA}$ & VERBINDUNG & 1 & 1 & NA \\
\hline rs19980408_1bvr168093.xml & Art. 14 Abs. 1 S. 1 & 14 & 1 & 1 & 1 NA & NA & NA & NA \\
\hline rs19980408_1bvr177396.xml & Art. 12 Abs. 1 & 12 & & $1 \mathrm{NA}$ & NA & NA & NA & NA \\
\hline
\end{tabular}

Abbildung 2: Festgestellte Verletzungen, Ausschnitt aus der Tabelle

Es kommen jedoch auch Verbindungen von drei Normen oder von mehreren Absätzen der gleichen Norm mit einer weiteren Norm vor. Unsere Regeln zur automatischen Annotation lassen nach dem „in Verbindung mit“ nur eine weitere Norm zu. Dem lag der Gedanke zugrunde, dass es mit maschinellen Mitteln schwierig zu beurteilen sein könnte, ob ein Ausdruck wie „... verletzt Art. A in Verbindung mit Art. B und Art. C ... “ bedeutet, dass Art. A mit Art. B verbunden wird und Art. C isoliert daneben steht oder ob Art. A mit Art. B und Art. C verbunden wird. Ausdrücke wie „Artikel 13 Absatz 1, 2 in Verbindung mit Artikel 19 Absatz 4“57, haben wir entsprechend unserem Vorgehen

48 Meinke (Fn. 41) S. 103 in Bezug auf BVerfGE 39, 1, 51 - Schwangerschaftsabbruch I und BVerfGE 46, 160 - Schleyer.

49 Breckwoldt (Fn. 42), S 58; Meinke (Fn. 41) S. 240.

50 Meinke (Fn. 41) S. 245.

51 Meinke (Fn. 41) S. 109, 223.

52 So bzgl. der Entscheidung BVerfGE 104, 337 zum Schächten Augsberg/Augsberg AöR 2007, 539, 547.

53 Meinke (Fn. 41) S. 168.

54 Meinke (Fn. 41) S. 252.

55 Breckwoldt (Fn. 42), S. $16 \mathrm{f}$.

56 Breckwoldt (Fn. 42), S. 18.

57 BVerfG, Beschluss der 3. Kammer des Zweiten Senats vom 22. Januar 2002 - 2 BvR 1473/01. 
bei unverbundenen Artikeln manuell in zwei Datensätze umgewandelt, also „Art. 13 Abs. 1 in Verbindung mit Art. 19 Abs. 4“ und „Art. 13 Abs. 2 in Verbindung mit Art. 19 Abs. 4“. Analog sind wir vorgegangen, wenn auf der rechten Seite der Verbindung mehrere Absätze vorhanden waren. Dies wird der Komplexität der Verbindungen möglicherweise nicht gerecht. Die maschinelle, quantitative Auswertung erfordert jedoch notwendigerweise eine gewisse Modellierung, welche mit Vereinfachungen einhergeht. Auf eine tiefergehende Theoriebildung, wann eine solche Verbindung ein eigenständiges Grundrecht darstellt, haben wir verzichtet, da sie zwangsläufig ein Lesen der einzelnen Entscheidungen voraussetzt, was gerade nicht Sinn einer (weitgehend) automatischen Auswertung ist. Die Verbindungspraxis des Bundesverfassungsgerichts könnte Gegenstand einer eigenen quantitativen Untersuchung sein. Weiterhin ist zu beachten, dass die Bedeutung eines Grundrechts auch im Rahmen der Prüfung eines anderen Grundrechts berücksichtigt werden kann, ohne dass klar wird, ob damit inhaltlich eine Kombination der Grundrechte intendiert ist. ${ }^{58}$ Diesen Fall können wir nicht erkennen.

Weiterhin gibt es einige Entscheidungen, die einen Tenorsatz enthalten, der aussagt, dass etwas „nichtig“, „unvereinbar“ oder „nicht vereinbar“ ist, bei denen aber im Tenor keine Verletzung einer Grundgesetznorm festgestellt wird, ${ }^{59}$ sowie Entscheidungen, in denen gar kein Tenor gefunden wurde. Diese haben wir überprüft und 25 weitere Entscheidungen manuell in die Tabelle aufgenommen.

Für die Untersuchung der gerügten Grundrechte sind wir ganz ähnlich vorgegangen. Es fällt auf, dass die gerügten Grundrechte weniger detailliert bzw. „standardisiert“ wiedergegeben sind, manche Rügen sind sehr pauschal. ${ }^{60}$ Es gibt häufiger lange Ketten von Grundrechten ${ }^{61}$ und es fehlen häufiger Absatz und Satz. Manchmal wird ein paar Sätze später die Rüge konkretisiert und es werden Absatz und Satz angegeben, so dass manche Rügen sowohl mit als auch ohne Absatz enthalten sind. Dies kann bei der Auswertung berücksichtigt werden. Da es auch insgesamt viel mehr Ergebnisse gab als bei den verletzten Grundrechten, waren hier ca. 200 Datensätze manuell umzuformen. Zusätzlich wurden, wie bereits erwähnt, auch typische Begriffe im Zusammenhang mit Grundrechtsrügen (z.B. „faires Verfahren“), die sich innerhalb einer Rüge befanden, ausgewertet, diesen war durch GATE schon das passende Zitat zugewiesen worden. Soweit dies mehrdeutig war (z.B. „Ungleichbehandlung“, „Bestimmtheitsgebot“) wurden diese ebenfalls manuell nach Abgleich mit den Entscheidungen ergänzt, soweit nicht ohnehin bereits ein Zitat gefunden wurde.

\section{Wie wir zählen}

Die so erstellten Datensätze wurden mithilfe von $\mathrm{R}$, einer Programmiersprache für Statistikanwendungen, ${ }^{62}$ ausgewertet. Dabei werden die Informationen aus der bereits erwähnten

$58 \mathrm{Heß,} \mathrm{Grundrechtskonkurrenzen,} \mathrm{2000,} \mathrm{S.} 86$ f.

59 Beispielsweise BVerfGE 10, 59, 60.

60 „Art. 1 bis 19 GG [...]“, BVerfG, Beschluss der 3. Kammer des Zweiten Senats vom 01. Juli 2002 - 2 BvR 578/02.

61 BVerfGE 77, 137, 138.

62 R Core Team, R: A language and environment for statistical computing, https://www.R-project.org/ , dabei verwendeten wir insbesondere die Pakete ggplot2 (Wickham, ggplot2: Elegant Graphics for Data Analysis, New-York 2016, https://ggplot2.tidyverse.org), plyr (ders., The Split-Apply-Combine Strategy for Data Analysis, Journal of Statistical Software, 40(1), 1, http://www.jstatsoft.org/v40/i01/) und stringr (ders., stringr: Simple, Consistent 
Metadaten-Tabelle (u.A. Aktenzeichen, Spruchkörper, Datum) mit einbezogen. Es werden alle Verfahren außer Acht gelassen, die keine Verfassungsbeschwerden sind, also fehlerhafterweise in die Tabelle aufgenommen wurden. In die Tabelle wurden teilweise auch Grundgesetznormen aufgenommen, die kein Grundrecht sind, daher entfernen wir alle Zeilen, in denen nicht mindestens ein „Grundrecht“ steht (ein Grundrecht kann also zunächst mit einem Nicht-Grundrecht verbunden sein). Als „Grundrecht“ verstehen wir die Artikel 1, 2, 3, 4, 5, 6, 7, 8, 9, 10, 11, 12, 12a, 13, 14, 16, 16a, 17, 19, 33, 38, 101, 102, ${ }^{63}$ 103, 104 sowie die Artikel 136-139, 141 der WRV64, 20 (Staatsstrukturprinzipien als Teil von Verbindungen), 21 (für Parteien) und 28 (Abs. 1). Wir betrachten somit ausdrücklich auch solche Vorschriften als „Grundrechte“ (was bereits die grundrechtsgleichen Rechte mit einschließt), die nicht im Katalog des Art. 93 Abs. 1 Nr. 4a GG aufgeführt sind, in der Spruchpraxis des Bundesverfassungsgerichts jedoch über Verbindungskonstruktionen etc. dergestalt subjektiviert sind, dass sie Verfassungsbeschwerden zum Erfolg verhelfen können. Datensätze mit Art. 28 Abs. 2 GG werden entfernt, da wir Kommunalverfassungsbeschwerden ausschließen wollen.

Schließlich werden doppelte Datensätze entfernt. Wir zählen also Grundrechte, deren Verletzung festgestellt wurde, nur einmal pro Entscheidung, unabhängig davon, wie viele Beschwerdeführer oder Verfahrensgegenstände in einer Verfassungsbeschwerde behandelt werden. Dadurch wird auch die Praxis, Verfahren zu verbinden, die jenseits des § 66 BVerfGG als allgemeines Prinzip des Prozessrechts zulässig ist, ${ }^{65}$ widergespiegelt. Es bleiben 2.745 Datensätze von verletzten Grundrechten übrig, darunter 1.038 aus Senats- und Plenums- ${ }^{66}$ und 1.707 aus Kammerentscheidungen. Insgesamt sind 842 verschiedene Senats- und 1.420 verschiedene Kammerentscheidungen erfasst. Mit unserer Herangehensweise nehmen wir in Kauf, dass wir nicht mehr erfassen können, ob sich die Verletzung auf den gleichen Gegenstand (und verschiedene Beschwerdeführerinnen) oder auf verschiedene Gegenstände bezieht. Wir berücksichtigen nur Verfassungsbeschwerden, aber nicht die Verletzungen von Grundrechten, die in Verfahren der konkreten oder abstrakten Normenkontrolle (sofern sie nicht mit einer Verfassungsbeschwerde verbunden sind) festgestellt werden. Die gerügten Grundrechte werden auf ähnliche Weise erfasst. Hier haben wir 12.644 Datensätze, davon 4.388 aus Senats- und 8.256 aus Kammerentscheidungen.

Wrappers for Common String Operations, R package version 1.3.1., https://CRAN.R-project.org/package=stringr) (jeweils letzter Abruf am 3. Dezember 2019).

63 Offen gelassen in BVerfGE 60, 348, 354.

64 Codiert als W136, W137, W138, W139, W141.

65 Bethge, in: Maunz/Schmidt-Bleibtreu/Klein/Bethge, Bundesverfassungsgerichtsgesetz, 57. EL Juni 2019 , § 66 Rn. 6.

66 Im Folgenden soll der Begriff „Senatsentscheidungen“ bzw. „-verfahren“ auch die wenigen Plenarentscheidungen umfassen. 


\section{Ergebnisse}

\section{Die am häufigsten verletzten Grundrechte (absolute Erfolgszahlen)}

Für einen groben Überblick interessiert uns zunächst das Vorkommen einzelner Grundrechtsvorschriften, nur auf Art. und Abs. genau, ohne Berücksichtigung der Verbindung (auch hier wird jede Vorschrift nur ein Mal pro Entscheidung gezählt). Diese einzelne Zählung unterliegt weniger den Verzerrungen, die dadurch entstehen, dass unsere detaillierte Zählweise und die Berücksichtigung von Verbindungen $\mathrm{zu}$ sehr vielen jeweils nur ein Mal gezählten „Grundrechten“ führt. Wie man erwarten würde, kommt bei den Senatsentscheidungen ${ }^{67}$ Art. 103 Abs. 1 GG am häufigsten vor, dicht gefolgt von Art. 3 Abs. 1 und Art. 2 Abs. 1 GG. In den Kammerentscheidungen sieht es allerdings schon anders aus: Art. 20 Abs. 3 GG steht auf dem 1. Platz und es folgen noch vier weitere Grundrechte, bevor Art. 103 Abs. 1 GG auftaucht (Tab. 1). Allerdings steht Art. 20 Abs. 3 GG, wenn er verletzt ist, nur in ca. $25 \%$ der Senats- und in 22,5\% der Kammerentscheidungen an der ersten Stelle einer Verbindung, in 75 \% bzw. 77,5 \% der Fälle aber an der zweiten Stelle, was in der Tendenz ebenfalls nicht überrascht.

Berücksichtigt man die Verbindungen, ergibt sich Folgendes: Das Recht auf rechtliches Gehör kommt bei den Senatsentscheidungen am häufigsten vor, mit einigem Abstand gefolgt von dem allgemeinen Gleichheitssatz und der Berufsfreiheit (Abb. 3). Bei den Kammerentscheidungen steht dagegen Art. 3 Abs. 1 GG knapp vor Art. 103 Abs. 1 GG, vor der Berufsfreiheit folgen noch das Gebot effektiven Rechtsschutzes (Art. 19 Abs. 4 GG) und Art. 2 Abs. 1 in Verbindung mit Art. 20 Abs. 3 GG (Abb. 4). $\mathrm{Zu}$ beachten ist, dass unsere detaillierte Unterteilung in Artikel, Absatz und Satz, dazu führt, dass Vorschriften mehrmals in der Rangliste auftauchen, wo eine menschliche Betrachterin möglicherweise nur eine Vorschrift angenommen hätte (z.B. Art. 19 Abs. 4 GG und Art. 19 Abs. 4 S. 1 GG). Dies ist möglicherweise nur unterschiedlichen Zitiervorlieben der einzelnen Richter und Mitarbeiterinnen geschuldet. Zudem finden sich bei den hier nicht abgebildeten, selteneren Zitaten Verbindungen mit den weiter vorne genannten Vorschriften, die sich teilweise aufgrund der oben beschriebenen separaten Zählweise, von Absätzen und Sätzen anhäufen und die ein menschlicher Leser möglicherweise ebenfalls anders einordnen würde. Insgesamt gibt es bei den Senatsentscheidungen 75 und bei den Kammerentscheidungen 41 Zitate, die nur ein einziges Mal vorkommen, die meisten davon sind Verbindungen. Dies überrascht nicht, zumal auch in der qualitativen Literatur bereits darauf hingewiesen wurde, dass manche Verbindungen „zufällig“ erschienen oder ihr Mehrwert gegenüber einer Verknüpfung mit „und“ oder einem Komma zweifelhaft sei. ${ }^{68}$

Bereits die elf am häufigsten verletzten Grundrechte (bis Art. 6 Abs. 1 GG) bestimmen einen Großteil der Verfahren: In 605 Senatsentscheidungen ist mindestens eines dieser elf Grundrechte verletzt, das entspricht ca. $71 \%$ aller erfolgreichen Senatsentscheidungen und ca. $17 \%$ aller Senatsentscheidungen. Von den zehn am häufigsten verletzten Grundrechten in

67 Da unser Korpus sämtliche Senatsentscheidungen, aber Kammerentscheidungen erst ab 1997 enthält, empfiehlt sich weitgehend eine getrennte Betrachtung.

68 Siehe dazu Meinke (Fn. 41) S. 250. 
Kammerentscheidungen ist in 870 Kammerentscheidungen mindestens eines verletzt, das entspricht ca. $61 \%$ aller erfolgreichen Kammerentscheidungen und ca. $15 \%$ aller Kammerentscheidungen.

Ein differenziertes Bild ergibt sich bei der Betrachtung der einzelnen Senate: Während im Ersten Senat die Berufsfreiheit etwas vor Art. 103 Abs. 1 GG liegt (Abb. 5), ist das Recht auf rechtliches Gehör im Zweiten Senat das mit großem Abstand am häufigsten als verletzt befundene Grundrecht (Abb. 6).

\section{Die am häufigsten gerügten Grundrechte}

Hier interessieren uns die gleichen Fragen wie hinsichtlich der verletzten Grundrechte. Nach dem Aussondern der Datensätze, die keine Verfassungsbeschwerden oder keine Grundrechte betreffen oder mehrfach vorkommen, verbleiben 12.644 Datensätze, davon 8.256 zu Kammer- und 4.388 zu Senatsverfahren.

Bei der Betrachtung der einzelnen Vorschriften ohne Berücksichtigung der Verbindungen (Tab. 2) fällt bereits auf, dass bei den Senatsentscheidungen Art. 16 Abs. 2 GG mit 18 Verletzungen auf Platz 13 der häufigsten Grundrechtsverletzungen, aber nur auf Platz 30 der gerügten Grundrechte (28 Rügen) steht. Dies deutet darauf hin, dass Art. 16 Abs. 2 GG ein - bezogen auf die Häufigkeit der Rüge - erfolgreicheres Grundrecht sein könnte. Bei den Kammerentscheidungen steht Art. 104 Abs. 1 GG mit 58 Verletzungen auf Platz 11 der verletzten, aber nur auf Platz 16 der gerügten Grundrechte (107 Rügen).

Unter Berücksichtigung von Grundrechtsverbindungen sind der allgemeine Gleichheitssatz, gefolgt vom Recht auf rechtliches Gehör die am häufigsten gerügten Grundrechte. In Senatsentscheidungen folgt auf Platz 3 die allgemeine Handlungsfreiheit (Abb. 7), in Kammerentscheidungen die Rechtsweggarantie des Art. 19 Abs. 4 GG (Abb. 8).

Art. 20 Abs. 3 GG kommt häufiger an erster Stelle einer Verbindung oder allein vor, nämlich in ca. 61 \% der Senats- und in ca. 45 \% der Kammerentscheidungen. Dies kann aber auch auf die teilweise recht langen Ketten von gerügten Grundrechten zurückzuführen sein, in denen das Rechtsstaatsprinzip u.U. einzeln am Ende steht, wo das Bundesverfassungsgericht vielleicht eher eine Verbindung einer anderen Grundrechtsnorm mit dem Rechtsstaatsprinzip annehmen würde. ${ }^{69}$

Die zehn häufigsten in Senatsentscheidungen gerügten Grundrechte (bis Art. 1 Abs. 1 GG) werden in 1.283 Senatsentscheidungen gerügt, das entspricht $81 \%$ aller Senatsentscheidungen, in denen Rügen identifiziert wurden oder $35 \%$ aller Senatsentscheidungen. Bei den Kammerentscheidungen werden die zehn am häufigsten gerügten Grundrechte in 2.528 Kammerentscheidungen gerügt, das entspricht 80\% der erfolgreichen Kammerentscheidungen und 45 \% aller Kammerentscheidungen.

69 Beispielsweise BVerfG, Beschluss der 3. Kammer des Ersten Senats vom 19. Juli 2010 - 1 BvR 1873/09 - Rn. 7 bzw. 10. 


\section{Veränderungen im Laufe der Zeit: Art. 103 Abs. 1 GG und die Einführung der Anhörungsrüge}

Nicht für alle Kombinationen von Artikel, Absatz, Satz und verbundenen Artikeln, die wir als einzelnes Grundrecht zählen, können wir die Entwicklung über die Zeit betrachten, die meisten Kombinationen kommen zu selten vor, um aufschlussreiche Ergebnisse zu liefern. Wir beschränken uns daher hier auf das Recht auf rechtliches Gehör. Auffällig ist, dass es ab 1986 nur noch einzelne und ab 2005 überhaupt keine Senatsentscheidungen mehr zu Art. 103 Abs. 1 GG gibt (Abb. 9).

Bei den Kammerentscheidungen sind weniger Auffälligkeiten festzustellen, sie decken aber auch nur einen vergleichsweise kurzen Zeitraum ab (Abb. 10). Im Jahr 2005, dem Jahr der Ausweitung der Anhörungsrüge auf alle Prozessordnungen, ${ }^{70}$ ist die Zahl der wegen Art. 103 Abs. 1 GG erfolgreichen Verfassungsbeschwerden (die aber auch aus früheren Jahren stammen können) auf dem tiefsten Stand, danach nehmen die erfolgreichen Fälle wieder zu, es gibt sogar einen sehr hohen Wert im Jahr 2012. Dies lässt Zweifel aufkommen, ob die Anhörungsrüge den erwünschten Erfolg gebracht hat. Allerdings sind sowohl der Median als auch der Mittelwert der in einem Jahr wegen Art. 103 Abs. 1 GG erfolgreichen Verfassungsbeschwerden zwischen den beiden Zeiträumen kleiner geworden (Tab. 3).

Auch die Anzahl der Rügen von Art. 103 Abs. 1 GG nimmt ab den 1980er Jahren sowohl in Senatensentscheidungen (Abb. 11) als auch in Kammerentscheidungen (Abb. 12) ab.

Bei den Kammerentscheidungen zeigt sich auf den ersten Blick kein dramatischer Unterschied zwischen der Anzahl der Rügen vor und nach 2005. Auch der Median und der Mittelwert der Rügen pro Jahr vor und nach 2005 unterscheiden sich kaum. Allerdings wirkt sich eine 2005 eingeführte prozessrechtliche Regelung möglicherweise nicht sofort aus, da auch Verfahren entschieden werden, die ggf. schon längere Zeit anhängig sind. Vergleicht man die Zahlen vor und nach 2009, ist die Verringerung von Median und Mittelwert schon etwas auffälliger (Tab. 4).

\section{Die relativ erfolgreichsten Grundrechte}

Betrachtet man auch einzelne Sätze und Verbindungen, steht Art. 16 Abs. 2 GG bei den Senatsentscheidungen auf Platz 17 der am häufigsten verletzten, aber nur auf Platz 39 der am häufigsten gerügten Grundrechte (11 Verletzungen bzw. 19 Rügen). Es ist anzunehmen, dass ein Grundrecht, das in der Rangfolge der verletzten Grundrechte höher steht als in der Rangfolge der gerügten Grundrechte, auch ein relativ erfolgreiches Grundrecht ist. Solche Grundrechte sind z.B. Art. 2 Abs. 1 i.V.m. Art. 20 Abs. 3 GG (+10) $)^{71}$, Art. 2 Abs. 1 i.V.m. Art. 1 Abs. 1 GG (+11), Art. 5 Abs. 1 S. 2 GG (+9) und Art. 5 Abs. 3 S. 1 GG (+10). Grundrechte, die in der Reihenfolge der verletzten Grundrechte deutlich weiter unten stehen als in der Reihenfolge der gerügten Grundrechte sind z.B. Art. 20 Abs. 3 GG (-9), Art. 1 Abs. 1 GG (-20), Art. 9 Abs. 3 GG (-9)

70 Gesetz über die Rechtsbehelfe bei Verletzung des Anspruchs auf rechtliches Gehör (Anhörungsrügengesetz) vom 9. Dezember 2004, BGBl. I 2004, S. 3220.

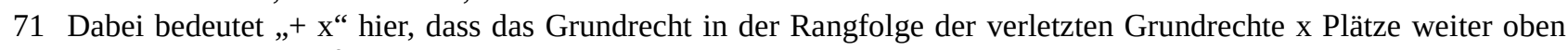
steht als in der Rangfolge der gerügten Grundrechte, „- X“ entsprechend, dass es x Plätze weiter unten steht. 
Bei den Kammerentscheidungen steht Art. 104 Abs. 1 GG über die Verbindung mit Art. 2 Abs. 2 S. 2 GG an 17. Stelle der verletzten und nur an 39. Stelle der gerügten Grundrechte (29 Verletzungen, 33 Rügen). Andere Grundrechte, die in der Rangfolge der verletzten Grundrechte höher stehen als in der Rangfolge der gerügten Grundrechte sind Art. 2 Abs. 1 i.V.m. Art. 1 Abs. 1 GG (+4) und Art. 5 Abs. 1 S. 1 GG (+10). Niedriger stehen dagegen Art. 101 Abs. 1 S. 2 GG (-5) oder Art. 14 Abs. 1 GG (14).

Einen genaueren Vergleich zwischen gerügten und verletzten Grundrechten erhalten wir, indem wir zunächst die Tabelle der gerügten Grundrechte mit der Tabelle der erfolgreichen Grundrechte verbinden, um die in einer konkreten Entscheidung erfolgreichen Rügen zu finden, und sodann die Anzahl für jedes erfolgreiche Grundrecht bzw. jede Verbindung ermitteln. Die „Erfolgsrate“ eines Grundrechts ist dann der Quotient aus erfolgreichen Rügen und Rügen insgesamt. Damit sehr seltene erfolgreiche Grundrechtsrügen nicht übermäßig ins Gewicht fallen, begrenzen wir die Auswertung auf Grundrechtsrügen, die mehr als fünfmal erfolgreich waren.

Unser Datensatz enthält 1.038 Verletzungen aus Senatsentscheidungen und 1.707 Verletzungen aus Kammerentscheidungen. Vergleichen wir das komplette Zitat eines Grundrechts, können nur 570 erfolgreiche Rügen aus Senatsentscheidungen (55 \%) und 1.033 erfolgreiche Rügen aus Kammerentscheidungen (60\%) berücksichtigt werden.

Das Ergebnis (Tab. 5) bestätigt die Annahme: Der Anteil an erfolgreichen Rügen ist in Senatsentscheidungen bei Art. 16 Abs. 2 GG besonders hoch (47 \%), aber auch bei Art. 5 Abs. 1 S. 1 GG (44 \%) und andere Zitate von Art. 5 GG stehen ebenfalls weit vorne. Für Art. 103 Abs. 1 GG beträgt der Anteil $40 \%$. Bei den Kammerentscheidungen liegt Art. 2 Abs. 2 S. 2 in Verbindung mit Art. 104 Abs. 1 S. 1 GG vorne (64 \%), gefolgt von Art. 12 Abs. 1 GG und Art. 6 Abs. 2 S. 1 GG. Art. 103 Abs. 1 GG steht erst auf Platz 19 mit $16 \%$.

Dieses Resultat muss unter Vorbehalt gesehen werden: Aufgrund unserer sehr differenzierten Zählung werden Angaben von Grundrechten mit und ohne Absatz und Satz als unterschiedliche Grundrechte gezählt, z.B. wird „Art. 5“ von „Art. 5 Abs. 1“ und „Art. 5 Abs. 1 S. 1“ getrennt gezählt, auch wenn vielleicht inhaltlich das gleiche gemeint ist. Es gibt in dieser Rechnung keinen Erfolg, wenn Art. 5 GG gerügt wurde, aber das Bundesverfassungsgericht sich genauer ausgedrückt hat, was häufig der Fall sein dürfte. 
Verletzung einzelner Grundrechte in Senatsentscheidungen

\begin{tabular}{l|r}
\hline \multicolumn{1}{|c|}{ Grundrecht } & Anzahl \\
\hline Art. 103 Abs. 1 & 177 \\
\hline Art. 3 Abs. 1 & 160 \\
\hline Art. 2 Abs. 1 & 139 \\
\hline Art. 12 Abs. 1 & 115 \\
\hline Art. 20 Abs. 3 & 77 \\
\hline Art. 19 Abs. 4 & 56 \\
\hline Art. 5 Abs. 1 & 50 \\
\hline Art. 1 Abs. 1 & 40 \\
\hline Art. 14 Abs. 1 & 37 \\
\hline Art. 6 Abs. 1 & 36 \\
\hline
\end{tabular}

Verletzung einzelner Grundrechte in Kammerentscheidungen

\begin{tabular}{|l|r|}
\hline \multicolumn{1}{|c|}{ Grundrecht } & Anzahl \\
\hline Art. 20 Abs. 3 & 278 \\
\hline Art. 3 Abs. 1 & 254 \\
\hline Art. 2 Abs. 1 & 253 \\
\hline Art. 19 Abs. 4 & 230 \\
\hline Art. 2 Abs. 2 & 206 \\
\hline Art. 103 Abs. 1 & 145 \\
\hline Art. 12 Abs. 1 & 107 \\
\hline Art. 5 Abs. 1 & 91 \\
\hline Art. 1 Abs. 1 & 85 \\
\hline Art. 6 Abs. 2 & 66 \\
\hline
\end{tabular}

Tabelle 1: Die 10 am häufigsten als verletzt bezeichneten Grundrechtsvorschriften (einschließlich Rechtsstaatsprinzip) ohne Berücksichtigung von Verbindungen

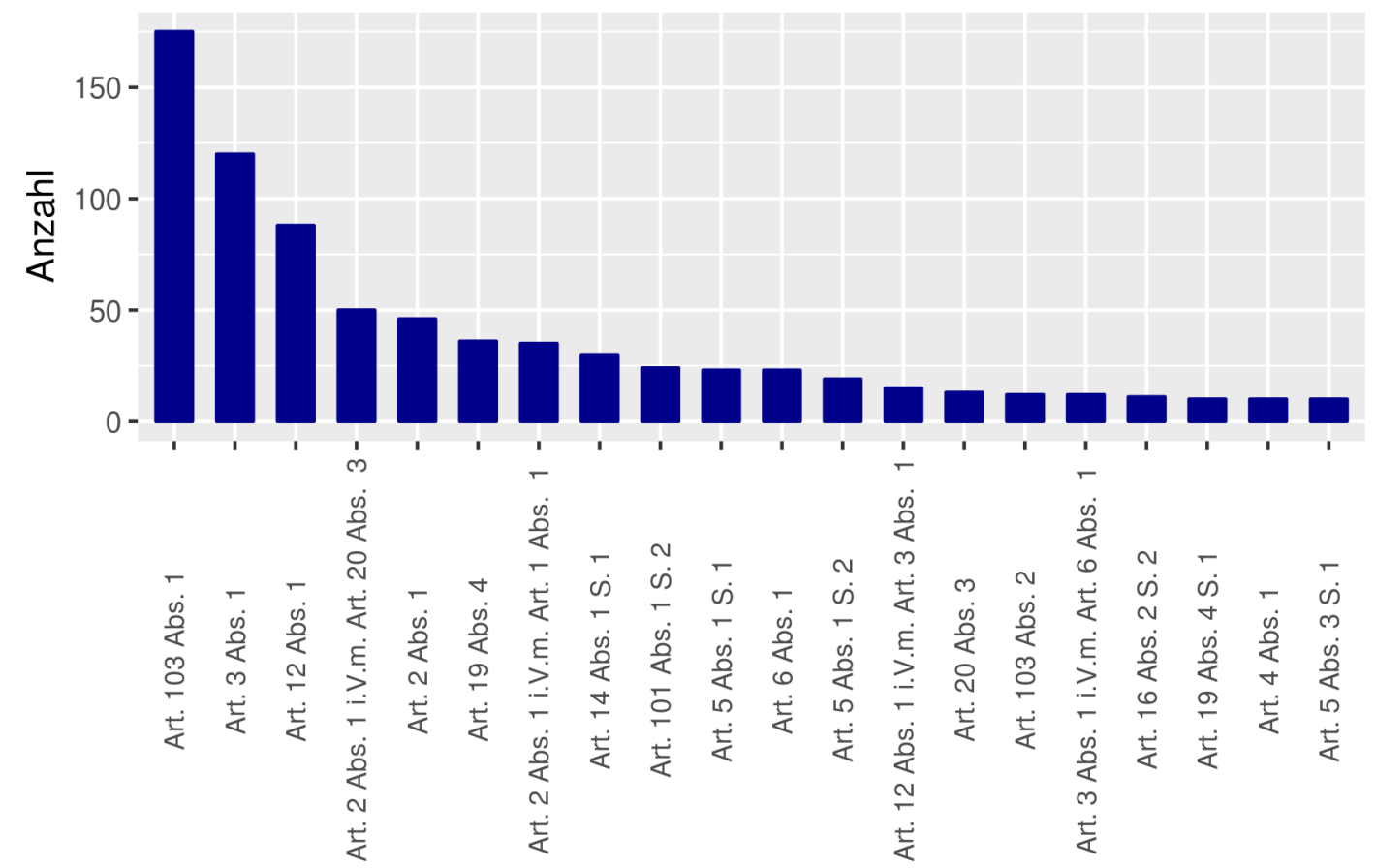

Abbildung 3: Die 20 am häufigsten verletzten Grundrechte in Senatsentscheidungen (min. 10 mal verletzt) 


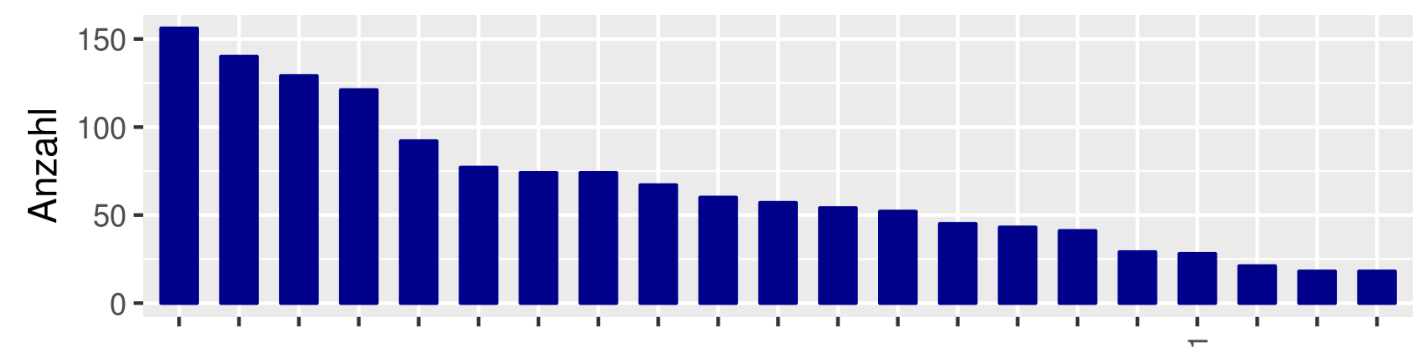

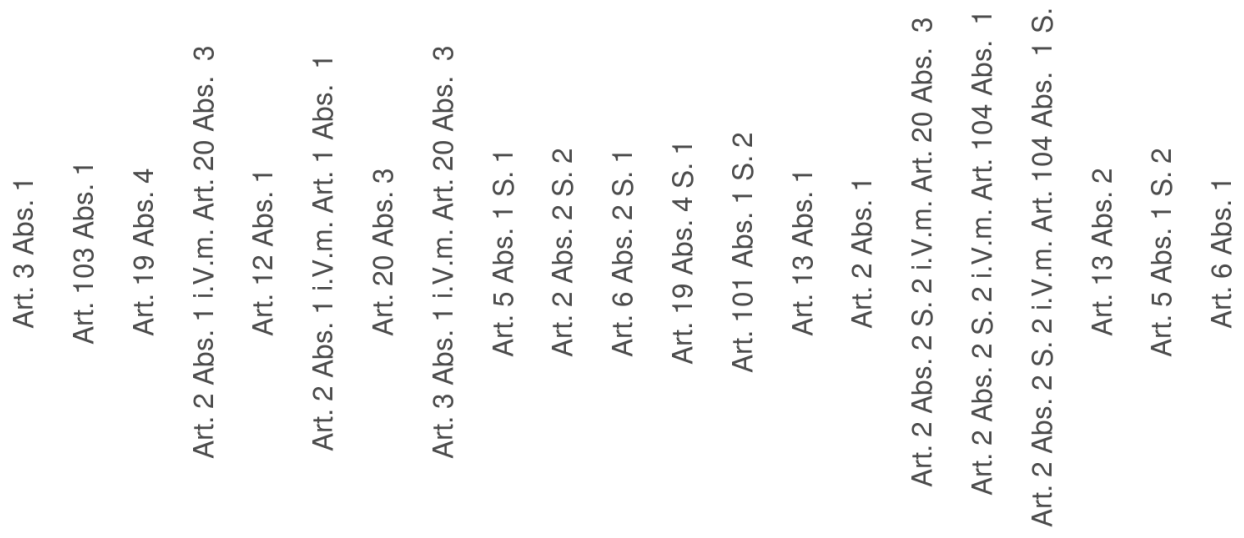

Abbildung 4: Die 21 am häufigsten verletzten Grundrechte in Kammerentscheidungen (ab 1998, min. 18 mal verletzt)

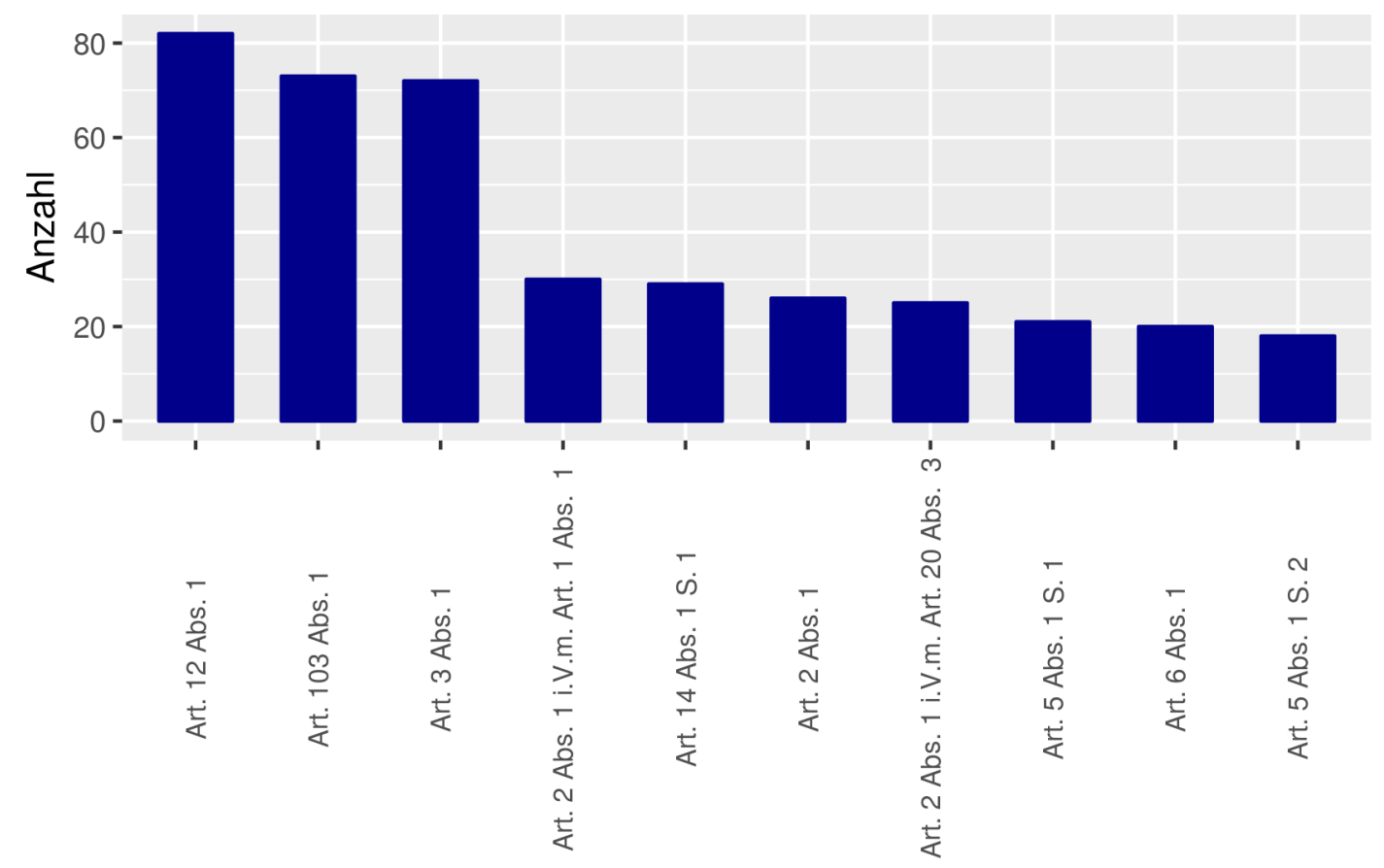

Abbildung 5: Die 10 am häufigsten verletzten Grundrechte in Entscheidungen des Ersten Senats (min. 18 mal verletzt) 


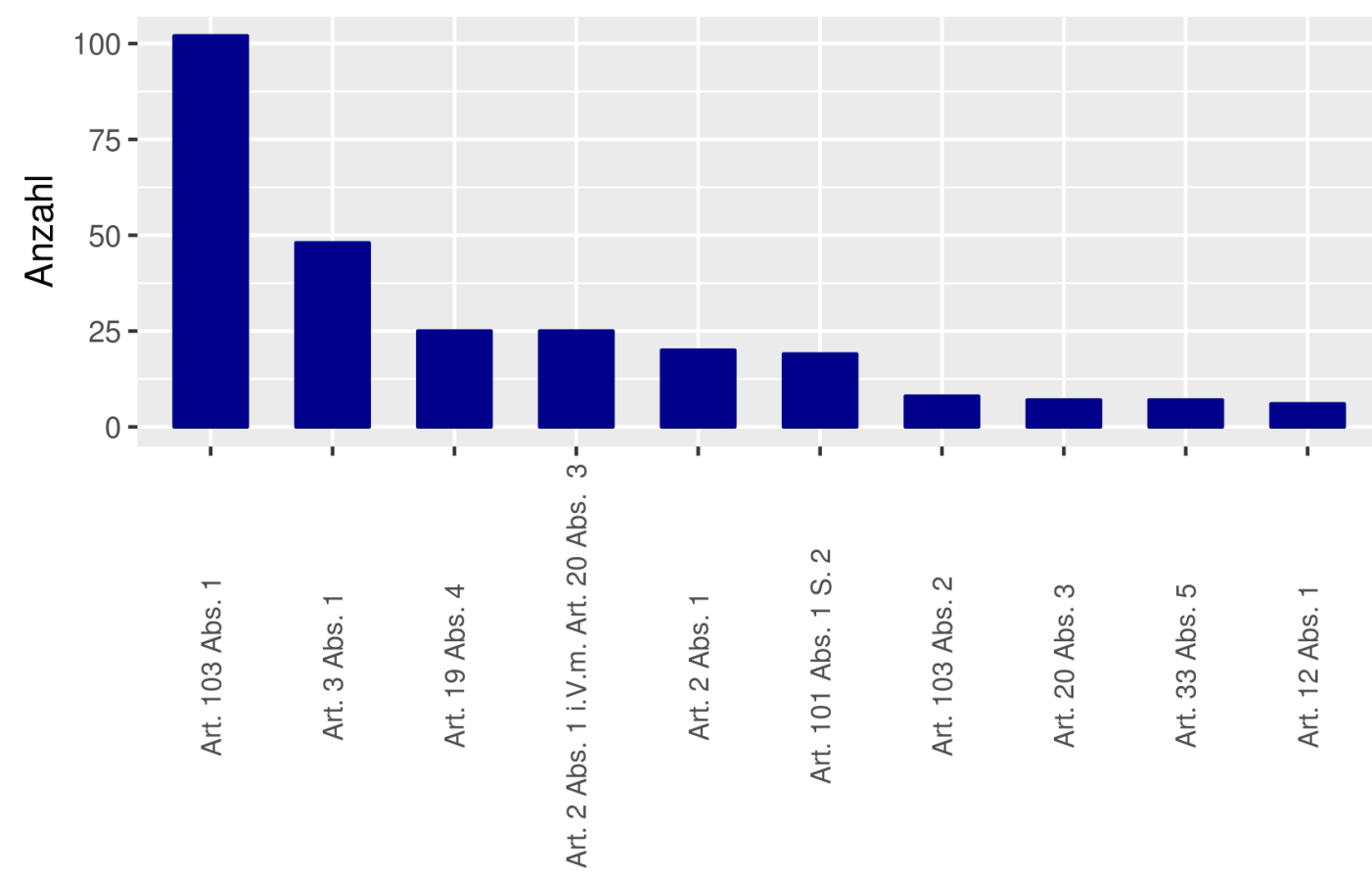

Abbildung 6: Die 10 am häufigsten verletzten Grundrechte in Entscheidungen des Zweiten Senats (min. 6 mal verletzt)

\begin{tabular}{|c|c|}
\hline \multicolumn{2}{|c|}{$\begin{array}{l}\text { Rügen einzelner Grundrechte in } \\
\text { Senatsentscheidungen }\end{array}$} \\
\hline Grundrecht & Anzahl \\
\hline Art. 3 Abs. 1 & 568 \\
\hline Art. 2 Abs. 1 & 448 \\
\hline Art. 103 Abs. 1 & 381 \\
\hline Art. 12 Abs. 1 & 233 \\
\hline Art. 20 Abs. 3 & 212 \\
\hline Art. 14 Abs. 1 & 177 \\
\hline Art. 19 Abs. 4 & 177 \\
\hline Art. 1 Abs. 1 & 156 \\
\hline Art. 3 & 152 \\
\hline Art. 14 & 141 \\
\hline Art. 5 Abs. 1 & 141 \\
\hline
\end{tabular}

\begin{tabular}{|c|c|}
\hline $\begin{array}{l}\text { Rügen einzeln } \\
\text { Kammeren }\end{array}$ & $\begin{array}{l}\text { chte in } \\
\text { jen }\end{array}$ \\
\hline Grundrecht & Anzahl \\
\hline Art. 3 Abs. 1 & 1241 \\
\hline Art. 2 Abs. 1 & 998 \\
\hline Art. 20 Abs. 3 & 814 \\
\hline Art. 103 Abs. 1 & 810 \\
\hline Art. 19 Abs. 4 & 663 \\
\hline Art. 12 Abs. 1 & 466 \\
\hline Art. 2 Abs. 2 & 406 \\
\hline Art. 101 Abs. 1 & 376 \\
\hline Art. 1 Abs. 1 & 372 \\
\hline Art. 14 Abs. 1 & 364 \\
\hline Art. 5 Abs. 1 & 213 \\
\hline
\end{tabular}

Tabelle 2: Die 11 häufigsten Grundrechtsrügen (ohne Berücksichtigung von Verbindungen) in Senatsentscheidungen (ab 1951) und in Kammerentscheidungen (ab 1998) 


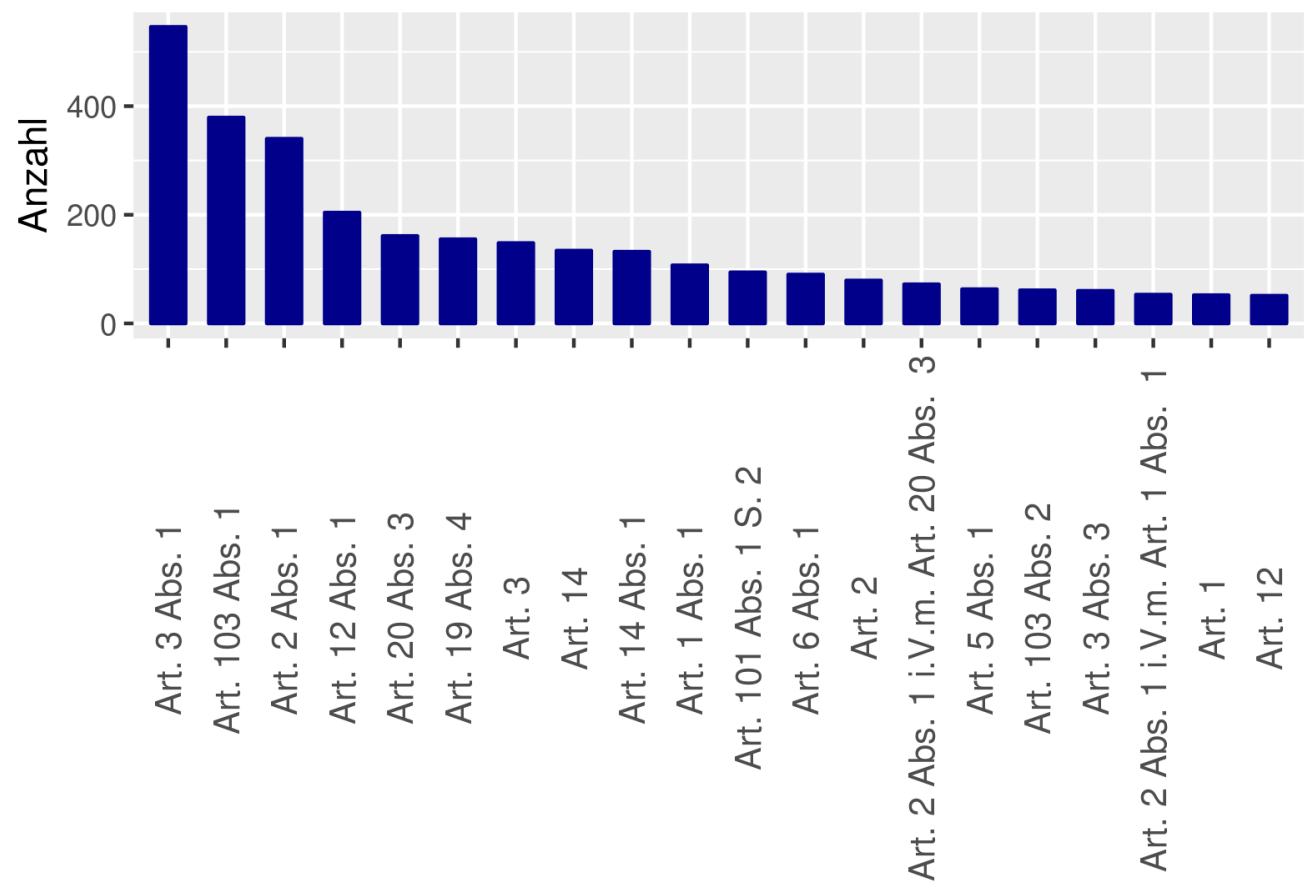

Abbildung 7: Die 20 am häufigsten gerügten Grundrechte in Senatsentscheidungen (min. 51 Rügen)

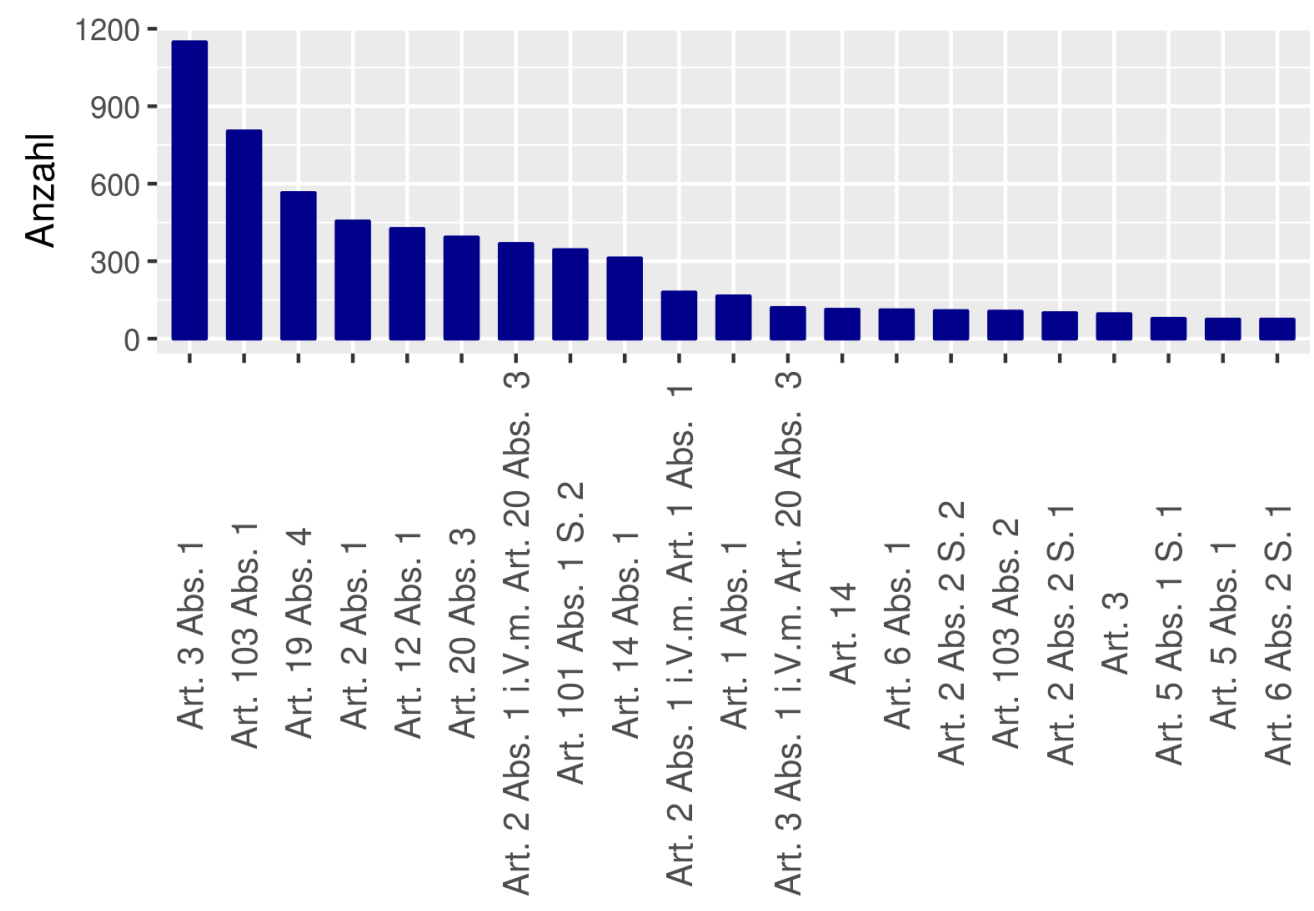

Abbildung 8: Die 21 am häufigsten gerügten Grundrechte in Kammerentscheidungen (ab 1998, min. 74 Rügen) 


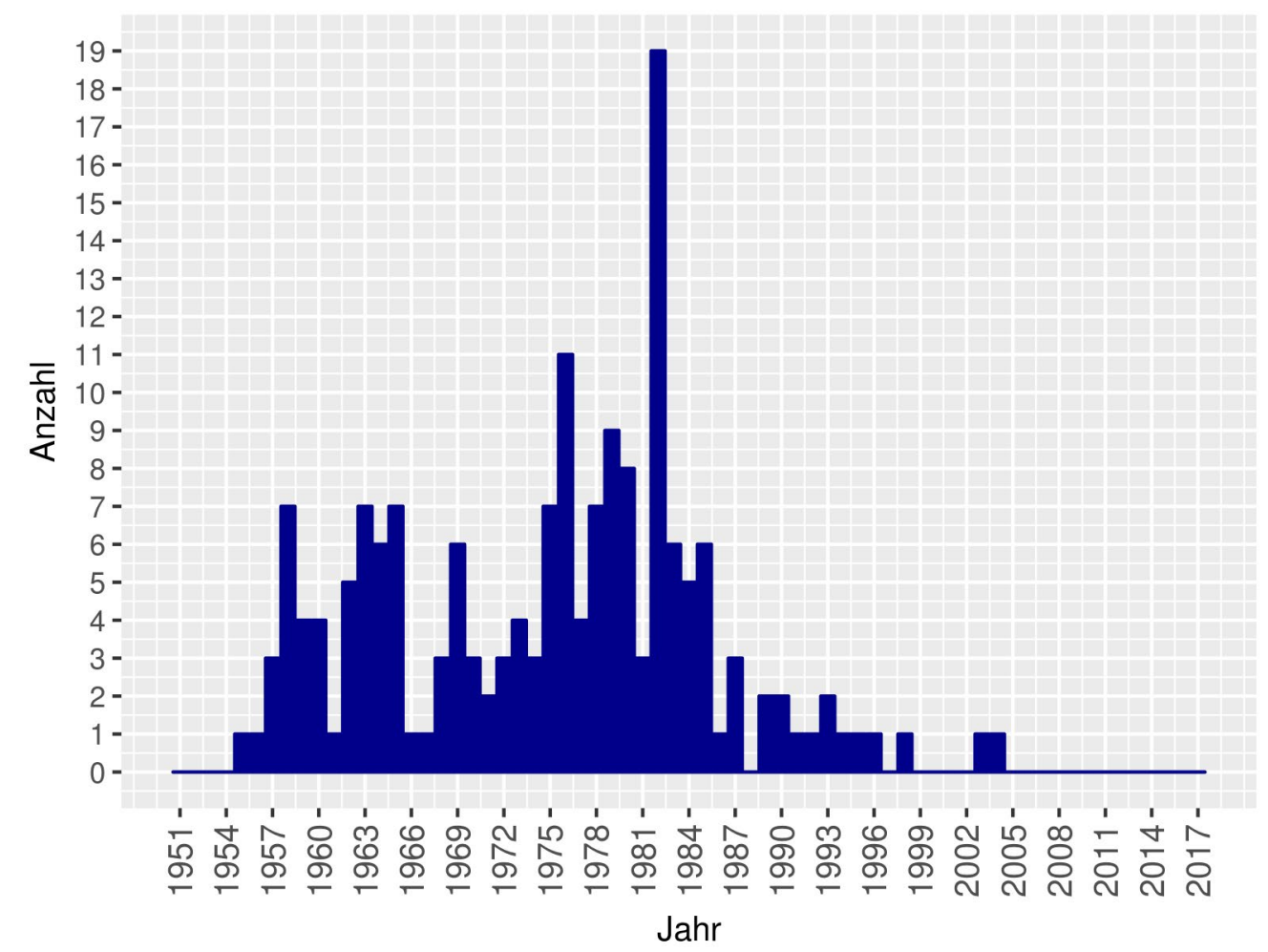

Abbildung 9: Verletzung von Art. 103 Abs. 1 GG, Senate, 1951-2017 


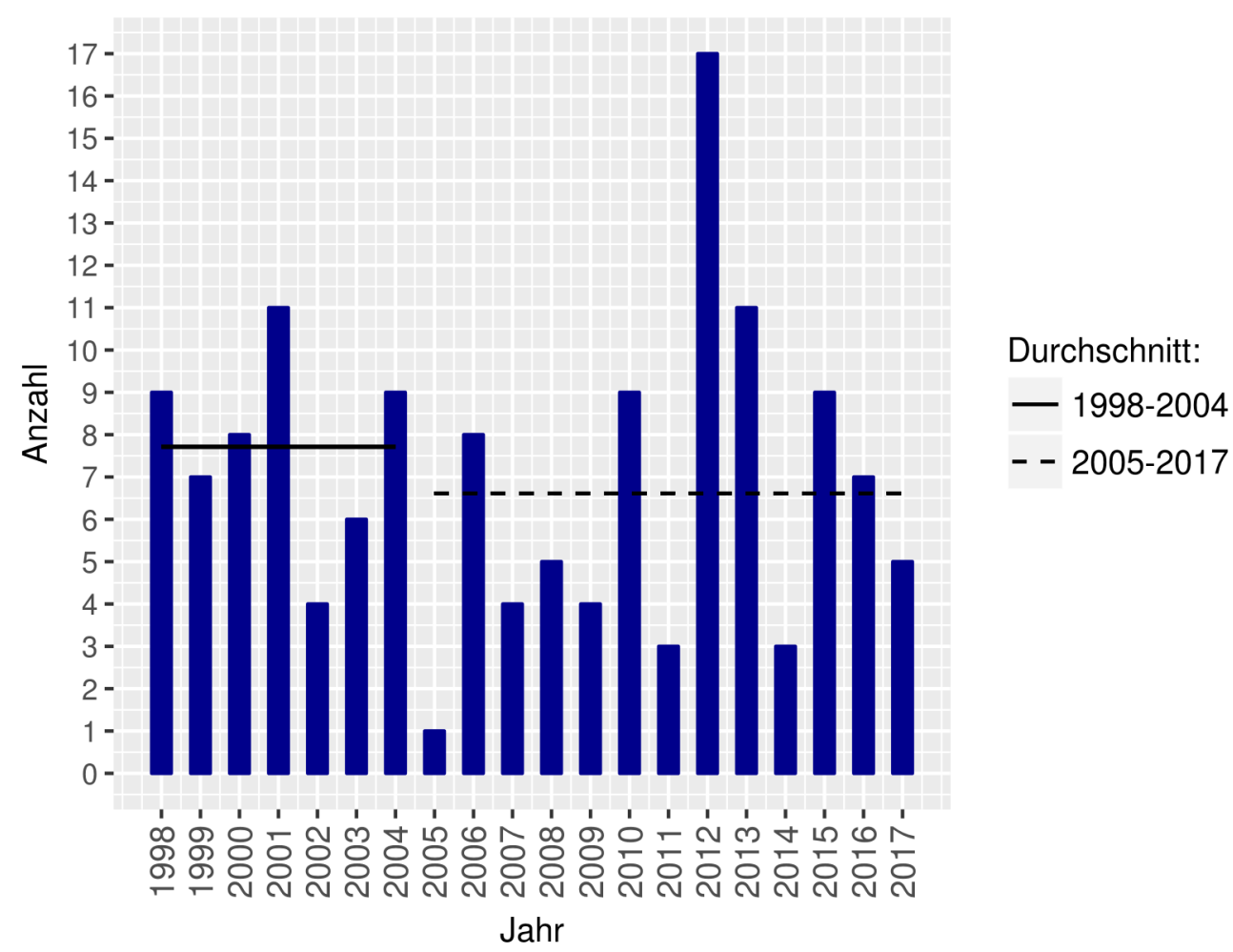

Abbildung 10: Verletzung von Art. 103 Abs. 1 GG, Kammern, 1998-2017

\begin{tabular}{|c|c|c|c|c|}
\hline \multicolumn{5}{|c|}{ Verletzung von Art. 103 Abs. 1 in den Kammerentscheidungen } \\
\hline & Minimum & Median & Mittelwert & Maximum \\
\hline 1998-2004 & 4 & 8 & 7,71 & 11 \\
\hline 2005-2017 & 1 & 5 & 6,62 & 17 \\
\hline
\end{tabular}

Tabelle 3: Minimum, Median, Mittelwert und Maximum vor und ab 2005 


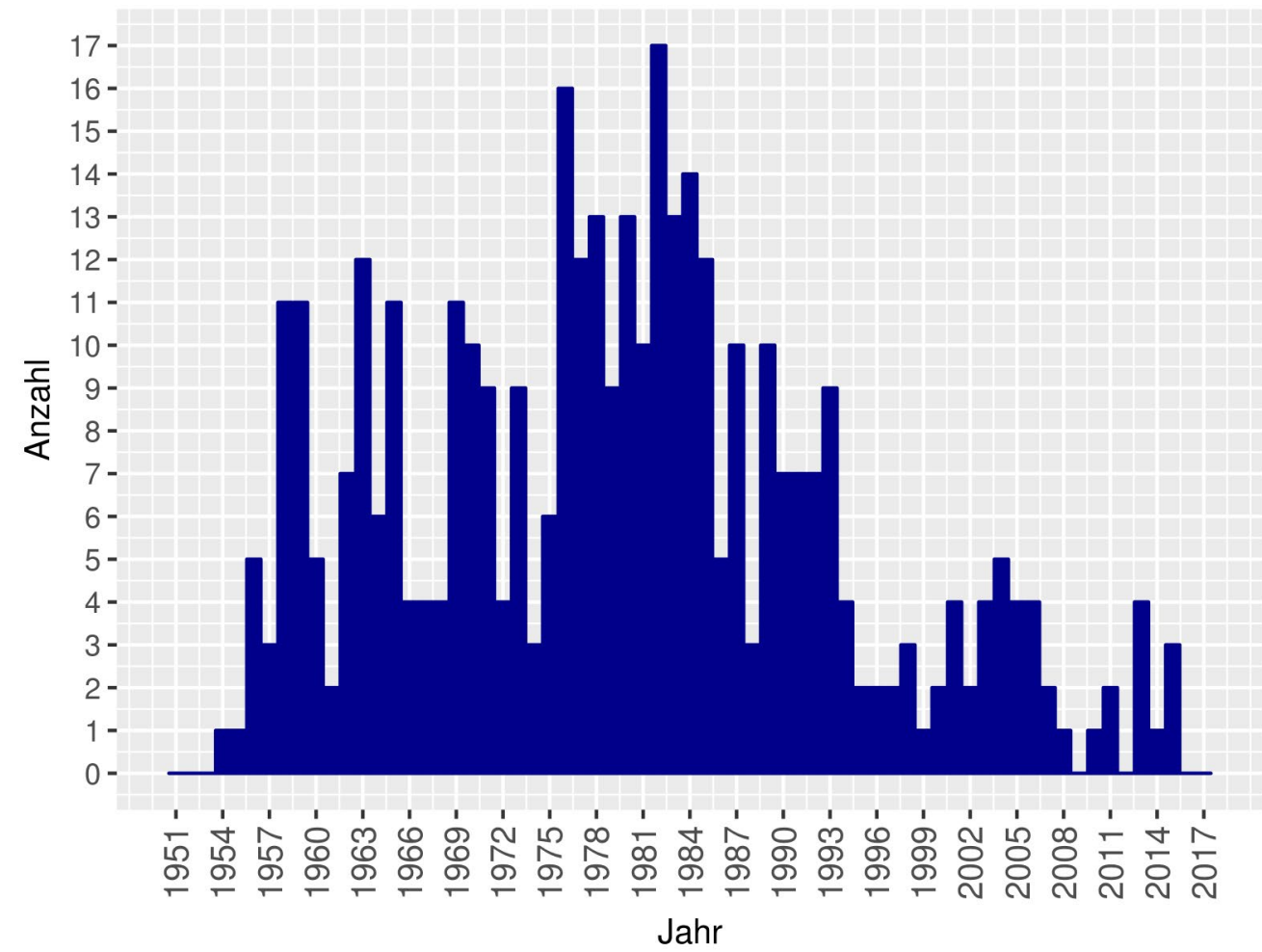

Abbildung 11: Anzahl der Rügen von Art. 103 Abs. 1 GG, Senate, 1951-2017

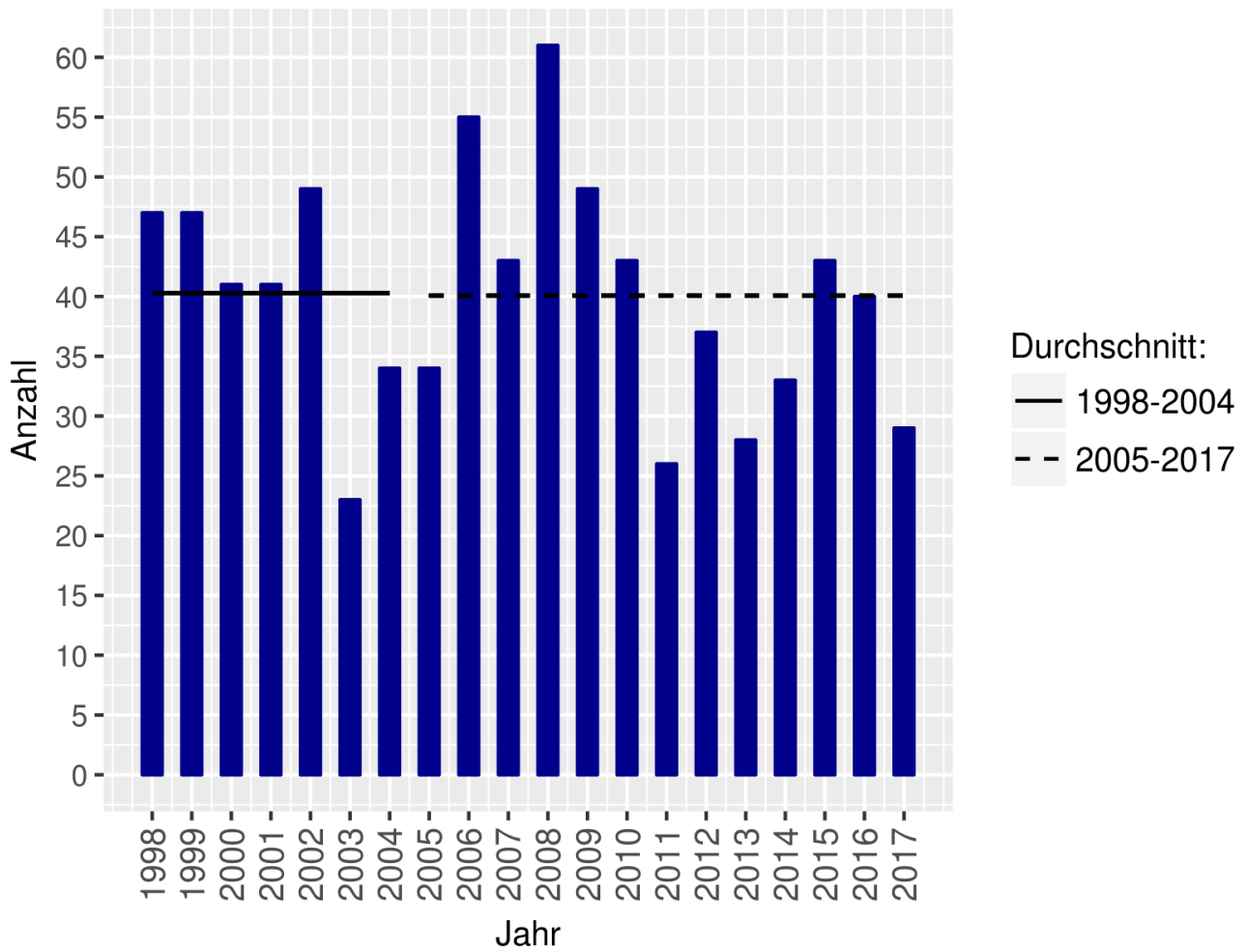

Abbildung 12: Anzahl der Rügen von Art. 103 Abs. 1 GG, Kammern, 1998-2017 
Rügen von Art. 103 Abs. 1 in den Kammerentscheidungen

\begin{tabular}{c|c|c|c|c|}
\hline & Minimum & Median & Mittelwert & Maximum \\
\hline $\mathbf{1 9 9 8 - 2 0 0 4}$ & 23 & 41 & 40,29 & 49 \\
\hline $\mathbf{2 0 0 5 - 2 0 1 7}$ & 26 & 40 & 40,08 & 61 \\
\hline $\mathbf{1 9 9 8 - 2 0 0 8}$ & 23 & 43 & 43,18 & 61 \\
\hline $\mathbf{2 0 0 9 - 2 0 1 7}$ & 26 & 37 & 36,44 & 49 \\
\hline
\end{tabular}

Tabelle 4: Minimum, Median, Mittelwert und Maximum vor und ab 2005 (weiß) bzw. vor und ab 2009 (grau) 
Anteil der erfolgreichen Rügen an allen Rügen (Senatsentscheidungen)

\begin{tabular}{c|l|r|r|r|}
\hline \multicolumn{1}{|c|}{ Gang } & \multicolumn{1}{|c|}{ Grundrecht } & freq.x & freq.y & Anteil \\
\hline 1 & Art. 16 Abs. 2 S. 2 & 9 & 19 & 0,47 \\
\hline 2 & Art. 5 Abs. 1 S. 1 & 11 & 25 & 0,44 \\
\hline 3 & Art. 103 Abs. 1 & 152 & 379 & 0,40 \\
\hline 4 & Art. 2 Abs. 1 i.V.m. Art. 1 Abs. 1 & 21 & 53 & 0,40 \\
\hline 5 & Art. 5 Abs. 3 S. 1 & 10 & 27 & 0,37 \\
\hline 6 & Art. 5 Abs. 1 S. 2 & 15 & 45 & 0,33 \\
\hline 7 & Art. 12 Abs. 1 & 53 & 204 & 0,26 \\
\hline 8 & Art. 4 Abs. 1 & 9 & 42 & 0,21 \\
\hline 9 & Art. 14 Abs. 1 S. 1 & 8 & 38 & 0,21 \\
\hline 10 & Art. 3 Abs. 2 & 6 & 29 & 0,21 \\
\hline 11 & Art. 6 Abs. 1 & 18 & 90 & 0,20 \\
\hline 12 & Art. 101 Abs. 1 S. 2 & 16 & 94 & 0,17 \\
\hline 13 & Art. 103 Abs. 2 & 87 & 61 & 0,16 \\
\hline 14 & Art. 3 Abs. 1 & 7 & 44 & 0,16 \\
\hline 15 & Art. 33 Abs. 5 & aller Rügen \\
\hline
\end{tabular}

Anteil der erfolgreichen Rügen an allen Rügen (Kammerentscheidungen)

\begin{tabular}{c|l|r|r|r|}
\hline Rang & \multicolumn{1}{|c|}{ Grundrecht } & freq.x & freq.y & Anteil \\
\hline 1 & Art. 2 Abs. 2 S. 2 i.V.m. Art. 104 Abs. 1 S. 1 & 9 & 14 & 0,64 \\
\hline 2 & Art. 12 Abs. 1 i.V.m. Art. 33 Abs. 2 & 6 & 10 & 0,60 \\
\hline 3 & Art. 6 Abs. 2 S. 1 & 38 & 74 & 0,51 \\
\hline 4 & Art. 5 Abs. 1 S. 1 & 39 & 77 & 0,51 \\
\hline 5 & Art. 2 Abs. 2 S. 2 i.V.m. Art. 20 Abs. 3 & 12 & 25 & 0,48 \\
\hline 6 & Art. 3 Abs. 1 i.V.m. Art. 20 Abs. 3 & 54 & 119 & 0,45 \\
\hline 7 & Art. 8 Abs. 1 & 9 & 20 & 0,45 \\
\hline 8 & Art. 13 Abs. 1 & 30 & 72 & 0,42 \\
\hline 9 & Art. 2 Abs. 2 S. 2 & 36 & 107 & 0,34 \\
\hline 10 & Art. 2 Abs. 2 S. 2 i.V.m. Art. 104 Abs. 1 & 11 & 33 & 0,33 \\
\hline 11 & Art. 16a Abs. 1 & 11 & 37 & 0,30 \\
\hline 12 & Art. 33 Abs. 2 & 14 & 52 & 0,27 \\
\hline 13 & Art. 19 Abs. 4 S. 1 & 16 & 62 & 0,26 \\
\hline 14 & Art. 2 Abs. 1 i.V.m. Art. 1 Abs. 1 & 44 & 179 & 0,25 \\
\hline 15 & Art. 5 Abs. 1 S. 2 & 14 & 57 & 0,25 \\
\hline 16 & Art. 13 Abs. 2 & 6 & 26 & 0,23 \\
\hline 17 & Art. 2 Abs. 1 i.V.m. Art. 20 Abs. 3 & 82 & 367 & 0,22 \\
\hline 18 & Art. 12 Abs. 1 & 80 & 425 & 0,19 \\
\hline 19 & Art. 103 Abs. 1 & 88 & 803 & 0,16 \\
\hline 20 & Art. 19 Abs. 4 & 564 & 0,16 \\
\hline & freq.x = Anzahl der erfolgreichen Rügen, freq.y = Anzahl aller Rügen & \\
\hline
\end{tabular}

Tabelle 5: Anteil der erfolgreichen Rügen an der Gesamtzahl der Rügen, Anteil >= $15 \%$ 


\section{Auswertung}

Diese Untersuchung ist nicht darauf ausgelegt, Kausalbeziehungen zu ermitteln. Auch aus mit statistischen Methoden ermittelten Korrelationen kann nur eine Aussage über Art und Stärke des Zusammenhangs von zwei Variablen getroffen werden, aber nicht zwangsläufig über die Kausalität der Veränderung einer Variablen für die Veränderung der anderen Variablen. ${ }^{72}$ Kausalität ist vielmehr eine Frage der inhaltlichen Interpretation, ${ }^{73}$ und setzt eine sorgfältige Experimentplanung voraus, ${ }^{74}$ die sich bezogen auf die Realität der eingehenden Verfassungsbeschwerden nicht bewerkstelligen lässt.

Im Folgenden sollen daher lediglich die Ergebnisse zusammengefasst, mögliche Interpretationsansätze vorgestellt werden und auf die bestehenden Einschränkungen hingewiesen werden.

\section{Eine erste Interpretation der Daten}

In Senatsentscheidungen ist das Recht auf rechtliches Gehör das am häufigsten verletzte Grundrecht. Bei den Kammerentscheidungen ist dagegen der allgemeine Gleichheitssatz etwas häufiger verletzt. Es folgen das Recht auf rechtliches Gehör und die Rechtsweggarantie des Art. 19 Abs. 4 GG. Jedenfalls im Zeitraum ab 1998 ist daher nicht mehr das Recht auf rechtliches Gehör, sondern der allgemeine Gleichheitssatz das am häufigsten verletzte Grundrecht. Das am häufigsten gerügte Grundrecht ist ebenfalls der allgemeine Gleichheitssatz noch vor dem Recht auf rechtliches Gehör, und zwar sowohl bei den Senats-, als auch bei den Kammerentscheidungen.

Sowohl in Senats- als auch in Kammerentscheidungen sind somit diejenigen Grundrechte besonders häufig verletzt, die einen weiten Schutzbereich haben und in vielen verschiedenen Situationen relevant werden können. So kann Art. 103 Abs. 1 GG potenziell in jedem Rechtsstreit relevant werden, das Gleiche gilt für Art. 19 Abs. 4 GG hinsichtlich des weiten Begriffs der öffentlichen Gewalt. Auch die weiter vorne platzierten Art. 3 Abs. 1 GG und Art. 2 Abs. 1 GG sind inhaltlich eher offen und können auf viele Lebenssachverhalte anwendbar sein; Art. 2 Abs. 1 i.V.m. Art. 20 Abs. 3 GG kann stellvertretend für verschiedenste Verfassungsverstöße stehen. Die ersten inhaltlich etwas stärker vorgeprägten Grundrechte in der Reihenfolge sind die Berufsfreiheit (Platz 3 der Senatsentscheidungen, Platz 5 der Kammerentscheidungen), die Eigentumsfreiheit (Platz 8 der Senatsentscheidungen) und die Meinungsfreiheit (Platz 10 der Senatsentscheidungen, Platz 9 der Kammerentscheidungen). Gerade die Berufsfreiheit kann allerdings ebenfalls von den unterschiedlichsten Rechtsakten betroffen sein.

Bei mehr als der Hälfte aller Verletzungen und in 60 - 70 \% aller Verfahren war ein Grundrecht aus der (kleinen) Gruppe der zehn am häufigsten verletzten Grundrechte bzw. Grundrechtsverbindungen verletzt. Die zehn am häufigsten gerügten Grundrechte werden sogar in 80 \% der Verfahren gerügt. Dies veranschaulicht, dass die verfassungsgerichtliche Praxis offenbar durch einige wenige

72 Meindl (Fn. 25) S. 220 f.; Zwerenz, Statistik - Einführung in die computergestützte Datenanalyse, 5. Aufl., 2011, S. $208 \mathrm{f}$.

73 Zwerenz (Fn. 72) S. 209.

74 Epstein/Martin, An Introduction to Empirical Legal Research, Oxford 2014, S. 6; Schäfer (Fn. 25), S. 37 ff. 
Grundrechte beherrscht wird, während andere, wie die Religionsfreiheit, die Versammlungsfreiheit oder das Recht auf Unverletzlichkeit der Wohnung, eher selten relevant werden.

Der Rückgang der Verletzungen von Art. 103 Abs. 1 GG in den späten 1980ern (Senatsentscheidungen) dürfte im Zusammenhang mit dem Gesetz zur Änderung des Gesetzes über das Bundesverfassungsgericht und zur Änderung des Deutschen Richtergesetzes vom 12. Dezember 1985 stehen. Durch dieses wurden die §§ 93b, 93c BVerfGG eingefügt, § 93a BVerfGG wurde neu gefasst. Die Kammern erhielten das Recht, Verfassungsbeschwerden stattzugeben. Es liegt nahe, dass dies dazu führte, dass mehr Beschwerden in Bezug auf Art. 103 Abs. 1 GG von den Kammern und weniger von den Senaten behandelt wurden. Allerdings fehlen uns die entsprechenden Zahlen für die Entwicklung der Kammerentscheidungen in der Zeit vor 1998.

Zwischen den Zeiträumen 1998-2004 (vor dem prozessordnungsübergreifenden Bestehen der Anhörungsrüge) und 2005-2017 ist zunächst nur ein leichter Rückgang der Verletzungen von Art. 103 Abs. 1 GG festzustellen. Das gleiche gilt für die Anzahl der Rügen von Art. 103 Abs. 1 GG. Allerdings wissen wir natürlich nicht, wie viele Verfassungsbeschwerden ohne Begründung aufgrund einer fehlenden Anhörungsrüge nicht zur Entscheidung angenommen wurden und auch die nicht angenommenen Verfassungsbeschwerden, die eine Begründung erhielten, könnten uns entgangen sein, wenn die Rüge von Art. 103 Abs. 1 GG nicht explizit erwähnt wird. Die inoffizielle Statistik, nach welcher die durchschnittliche Anzahl der auf Art. 103 Abs. 1 GG gestützten Verfassungsbeschwerden pro Jahr nach 2005 um fast 35 \% gesunken sein soll, ${ }^{75}$ können wir daher weder stützen noch widerlegen. Jedenfalls legen unsere Ergebnisse nahe, dass sich ein so starker Rückgang (noch) nicht in den Veröffentlichungen niedergeschlagen hat.

Das Recht auf rechtliches Gehör ist jedenfalls unter den Senatsentscheidungen ein Grundrecht, das relativ häufig zum Erfolg führt, was insbesondere für die Zeit vor 1986 gilt. Allerdings sind der Auslieferungsschutz des Art. 16 Abs. 2 S. 2 GG und die Meinungsfreiheit noch erfolgreicher. Bei den Kammerentscheidungen ist Art. 103 Abs. 1 GG erst weiter hinten in der Rangliste zu finden. Spätestens seit 1998 ist Art. 103 Abs. 1 GG also kein besonders erfolgreiches Grundrecht mehr. Stattdessen liegen das Recht auf Freiheit der Person in Verbindung mit den Garantien bei Freiheitsentziehung, die Berufsfreiheit, das elterliche Erziehungsrecht und die Meinungsfreiheit vorn. Dass sich die erfolgreichen Grundrechte so stark von den gerügten Grundrechten unterscheiden, ${ }^{76}$ ist nicht überraschend: Sowohl Art. 16 Abs. 2 GG als auch Art. 2 Abs. 2 S. 2 i.V.m. Art. 104 Abs. 1 S. 1 GG knüpfen an sehr spezielle Situationen an (Auslieferung bzw. Inhaftierung), so dass eine Rüge „ins Blaue hinein“ eher unwahrscheinlich erscheint - anders als bei Art. 3 Abs. 1 GG, Art. 2 Abs. 1 GG oder dem Rechtsstaatsprinzip. Die vergleichsweise hohe Platzierung von Art. 5 Abs. 1 S. 1 GG könnte auch die besondere Bedeutung dieses Grundrechts als „für die freiheitlich-demokratische Staatsordnung [...] schlechthin konstituierend“"77 widerspiegeln.

75 Siehe Nolte, in: v. Mangoldt/Klein/Starck (Fn. 15), Art. 103 Abs. 1 Fn. 4.

76 Dazu auch Bryde, Verfassungsentwicklung, 1982, S. 162; Heun, Der Zugang zum Bundesverfassungsgericht Zugangsfilter, Steuerungsmöglichkeiten des Gerichts, Mobilisierung, in: ders., Verfassung und Verfassungsgerichtsbarkeit im Vergleich, 2014, S. 144.

77 BVerfGE 7, 198, 208. 
Als Nebenprodukt unserer Untersuchung konnten wir auch Erkenntnisse über die Unterschiede zwischen Senaten und Kammern und zwischen den beiden Senaten gewinnen. Dass das Recht auf rechtliches Gehör die Rangliste der am häufigsten verletzten Grundrechte im Zweiten Senat anführt, aber im Ersten Senat noch hinter der Berufsfreiheit liegt, ist sicherlich darauf zurückzuführen, dass der Erste Senat nach dem Gesetz der primär für Verfassungsbeschwerden (und damit für Grundrechte) zuständige Senat ist ( $§ 14$ Abs. 1 BVerfGG), und dem Zweiten Senat nur für einzelne Rechtsbereiche die Zuständigkeit durch Plenumsbeschluss übertragen wurde. Da Fragen bzgl. des Rechts auf rechtliches Gehör offenbar kein eigenes „Rechtsgebiet“ darstellen, sondern entsprechend dem Rechtsgebiet, in dessen Kontext sie auftreten, behandelt werden, ${ }^{78}$ und dem Zweiten Senat die Zuständigkeit für alle nicht dem Ersten Senat zugeordneten Bereiche der Zivilgerichtsbarkeit ${ }^{79}$ sowie für weite Teile des Straf-, Strafverfahrens- und Strafvollzugsrechts ${ }^{80}$ zugewiesen sind, stellen sie möglicherweise eine relativ große Gruppe innerhalb der vergleichsweise wenigen vom Zweiten Senat behandelten Grundrechtsrügen dar.

Die unterschiedliche Reihenfolge der häufigsten Grundrechtsverletzungen in Senats- und Kammerentscheidungen legt nahe, dass manche Grundrechte eher in den Kammern behandelt werden, z.B. Art. 19 Abs. 4 GG, der Platz 3 bei den Kammer-, aber Platz 6 bei den Senatsentscheidungen belegt, andere dagegen eher in den Senaten, z.B. Art. 14 Abs. S. 1 GG, der Platz 8 bei den Senats- und Platz 22 bei den Kammerentscheidungen belegt.

Möglicherweise wirft die Eigentumsfreiheit mehr Fragen von grundsätzlicher verfassungsrechtlicher Bedeutung auf, und wird daher tendenziell häufiger von den Senaten behandelt als andere Grundrechte, jedenfalls bei absehbarem Erfolg, denn Art. 14 Abs. 1 GG steht immerhin auf Platz 9 der häufigsten Rügen in Kammerentscheidungen. Die Rechtsweggarantie des Art. 19 Abs. 4 GG scheint hingegen ein eher für Kammerentscheidungen prädestiniertes Grundrecht zu sein, was darauf zurückzuführen sein könnte, dass Art. 19 Abs. 4 GG, ähnlich wie Art. 103 Abs. 1 GG, in vielen verschiedenen Situationen relevant werden kann und die dabei zu entscheidenden Fragen eher kleinteilig sind. Dass Art. 103 Abs. 1 GG in den Kammerentscheidungen von Art. 3 Abs. 1 GG „überholt“ wurde, könnte auch daran liegen, dass die wichtigsten Fragen zu Art. 103 Abs. 1 GG bereits geklärt waren, bevor die Kammern ihre heutigen Befugnisse erhielten. Auch kommt es zu inhaltlichen Überlappungen, so dass gerade zwischen diesen beiden Grundrechten nicht immer ganz trennscharf unterschieden werden kann. ${ }^{81}$

\section{Einschränkungen}

Die Untersuchung war auf die Senatsentscheidungen und die ab 1998 veröffentlichten Kammerentscheidungen beschränkt. Als computergestützter Ansatz setzte sie eine konkrete

78 Dafür spricht, dass im Geschäftsverteilungsplan des 1. Senats geregelt ist, dass zu den aufgeführten Sachgebieten auch Verfahren gehören, in denen Rügen aus (z.B.) Art. 103 Abs. 1 GG überwiegen, während der Geschäftsverteilungsplan des 2. Senats dazu schweigt.

79 Siehe Punkt A. III. Beschluss des Plenums des Bundesverfassungsgerichts vom 24. November 2015 gemäß § 14 Abs. 4 des Gesetzes über das Bundesverfassungsgericht, zuletzt geändert durch Beschluss vom 21. November 2017.

80 Siehe Punkt A. I. Beschluss des Plenums des Bundesverfassungsgerichts vom 24. November 2015 gemäß 14 Abs. 4 des Gesetzes über das Bundesverfassungsgericht.

81 Siehe nur Remmert, in: Maunz/Dürig (Fn. 7) Art. 103 Abs. 1 Rn. 35 f. 
Modellbildung voraus und es ist davon auszugehen, dass nicht genau die gleichen Ergebnisse gefunden wurden, die ein menschlicher, juristisch ausgebildeter Leser gefunden hätte, hätte er alle 9.261 Dokumente selbst gelesen. Die Ermittlung der verletzten Grundrechte dürfte aufgrund der Standardisierung des Tenors relativ genau sein, allerdings fehlen uns Informationen zu den Kammerentscheidungen vor 1998. Die Ergebnisse zu der Frage, welche Grundrechte am häufigsten gerügt werden, sind dagegen mit Vorsicht zu genießen: Wir können von vornherein nur eine Auswahl der Verfassungsbeschwerden betrachten, nämlich die veröffentlichten. Was in den nichtveröffentlichten Verfassungsbeschwerden gerügt wurde, wissen wir nicht. Aber auch in den veröffentlichten ist nicht immer ausdrücklich ein gerügtes Grundrecht genannt, sei es, weil es angesichts des mangelnden Erfolgs den Richterinnen nicht wichtig schien, diese im „Tatbestand“ zu erwähnen, sei es, weil das Anliegen an sich für zu abwegig befunden wurde. ${ }^{82}$ Weiterhin ist die Art und Weise, wie die Rügen vorgebracht werden, sehr uneinheitlich, weswegen unser Annotationsalgorithmus nicht sämtliche Rügen erfasst und es häufiger zu Fehlern ${ }^{83}$ kommt.

\section{Fazit}

Diese Untersuchung hat die bislang eher anekdotischen Erkenntnisse zu Art. 103 Abs. 1 GG als dem am häufigsten gerügten und am häufigsten verletzten Grundrecht quantitativ und mit aktuellen Daten überprüft. Trotz einiger Einschränkungen führt sie zu relevanten Ergebnissen: Das Recht auf rechtliches Gehör kann nicht mehr ohne Weiteres als das am häufigsten gerügte und am häufigsten verletzte Grundrecht angesehen werden. In jüngerer Zeit steht dieser Titel dem allgemeinen Gleichheitssatz zu. Ein etwaiger Rückgang der Anzahl der auf Art. 103 Abs. 1 GG gestützten Verfassungsbeschwerden nach 2005 lässt sich bei Berücksichtigung allein der veröffentlichten Entscheidungen nicht ohne Weiteres bestätigen. Die relative Erfolgsquote von Art. 103 Abs. 1 GG ist nur bei den (älteren) Senatsentscheidungen mit $40 \%$ recht hoch. Andere Grundrechte wie der Auslieferungsschutz, die Rechtsgarantien bei Freiheitsentzug oder die Meinungsfreiheit weisen eine deutlich höhere Erfolgsquote auf. Während in absoluten Zahlen gesehen eher ,allgemein“ gehaltene Grundrechte zum Erfolg führen, sind es bei den relativen Erfolgsquoten gerade die spezielleren Grundrechte. Damit konnten die hier eingesetzten quantitativen und computergestützten Methoden Erkenntnisse hervorbringen, die für eine menschliche Leserin nicht offensichtlich sind und damit einen neuen Beitrag zur verfassungsrechtswissenschaftlichen Diskussion leisten.

82 Dies war möglicherweise der Fall in BVerfG, Beschluss der 2. Kammer des Zweiten Senats vom 16. Dezember 2016 - 2 BvR 349/16.

83 Z.B. ein Eintrag für eine Verletzung von „Art. 2 Abs. 9“. 